University of Louisville

ThinkIR: The University of Louisville's Institutional Repository

Electronic Theses and Dissertations

$12-2013$

\title{
Using the virtual world of Second Life to teach cultural competence for health science professional students.
}

Ashley Mack 1986-

University of Louisville

Follow this and additional works at: https://ir.library.louisville.edu/etd

\section{Recommended Citation}

Mack, Ashley 1986-, "Using the virtual world of Second Life to teach cultural competence for health science professional students." (2013). Electronic Theses and Dissertations. Paper 875.

https://doi.org/10.18297/etd/875

This Master's Thesis is brought to you for free and open access by ThinkIR: The University of Louisville's Institutional Repository. It has been accepted for inclusion in Electronic Theses and Dissertations by an authorized administrator of ThinkIR: The University of Louisville's Institutional Repository. This title appears here courtesy of the author, who has retained all other copyrights. For more information, please contact thinkir@louisville.edu. 


\title{
USING THE VIRTUAL WORLD OF SECOND LIFE TM TO TEACH CULTURAL COMPETENCE FOR HEALTH SCIENCE PROFESSIONAL STUDENTS
}

\author{
By
}

Ashley Mack

B.S., Kentucky State University, 2011

\begin{abstract}
A Thesis
Submitted to the Faculty of the School of Medicine of University of Louisville in Partial Fulfillment of the Requirements for the Degree of
\end{abstract}

Masters of Science

Anatomical Science and Neurobiology Department University of Louisville Louisville, KY

December 2013 



\title{
USING THE VIRTUAL WORLD OF SECOND LIFE TM TO TEACH CULTURAL COMPETENCE FOR HEALTH SCIENCE STUDENTS
}

\author{
By
}

\section{Ashley Mack}

B.S., Kentucky State University, 2011

A Thesis Approved on

November 22, 2013

By the Following Thesis Committee:

Jennifer Brueckner, Ph.D.

Nicole Herring, Ph.D.

April Richardson, Ph.D. 


\section{DEDICATION}

This thesis is dedicated to mother, Alpha Johnson, who always told me to strive for excellence. I wouldn't be where I am today without her. Also, to the entire Mack and Livers family who have prayed for me, encouraged me, and supported me throughout my entire matriculation. Lastly, I dedicate this thesis to Clevon

Pickett who has been a huge supporter to me throughout this entire experience. I love you all. Thank you! 


\section{ACKNOWLEDGMENTS}

I would like to acknowledge my mentor, Dr. Jennifer Brueckner. She has provided me with an invaluable amount of support, patience, and guidance. I have gained a wealth of knowledge from Dr. Brueckner and I thank her sincerely. I also would like to thank my other committee members, Dr. Nicole Herring and Dr. April Richardson for their time, patience, and direction over the past three years. Also many thanks to Dr. Charles Hubscher, the dean of the graduate program, who has been there to answer every question, reply to countless emails, and answer phone calls on my behalf. Lastly, but certainly not least, I would like to thank my mentor, Dr. Shorye Payne. You are an inspiration and you have been with me every step of the way pushing me towards my goal. Although the path I chose was not the one we mapped out three years ago, I am so thankful that now that I have reached one goal and I'm striving towards the next one, you're right here with me to celebrate. Thank you! 


\section{ABSTRACT \\ USING THE VIRTUAL WORLD OF SECOND LIFE TM TO TEACH CULTURAL COMPETENCE FOR HEALTH SCIENCE STUDENTS Ashley Mack}

November 22, 2013

Purpose: This study provides preliminary evidence that the use of technology, particularly the virtual world of Second Life ${ }^{\mathrm{TM}}$, can break down barriers by enhancing student interest in and satisfaction with the content. Methods: A preconference survey was administered to students attending the University of Louisville Cultural Competency Workshop series; six questions examined students' attitudes toward the LGBT community. Two health care scenarios were presented to students attending the LGBT sessions, either the traditional workshop setting, or a virtual Second Life ${ }^{\mathrm{TM}}$ session. A post-session survey was administered to students in the LGBT breakout sessions. Results: Using Second Life ${ }^{\mathrm{TM}}$ to present the LGBT health care scenarios significantly enhanced students' self reported sensitivity and compassion for LGBT patients and awareness of disparate health problems in the LGBT population. Conclusion: Second Life is an effective tool to enhance student interest and mastery of the sensitive nature of LGBT disparities in the healthcare industry. 
TABLE OF CONTENTS

Acknowledgments ___ iv

Abstract__ v

List of Figures __ vii

Introduction

Methods and Materials __ 29

Results — 49

Discussion _ 57

Conclusion__ 76

References _ 77

Appendices _ 85

Curriculum Vitae _ 116 


\section{LIST OF FIGURES}

FIGURE

PAGE\#

1. Cultural Competency Brochure 85

2. Hospital Screenshot from the Emergency Department Scenario 87

3. Doctor's Office Screenshot from the Office Visit Scenario 88

4. Second Life ${ }^{\mathrm{TM}}$ User Brochure 89

5. Logitech Headphone 103

6. Post-survey questionnaire 104

7. Pre-Session Survey Results: Question 5 106

8. Pre-Session Survey Results: Question 8 107

9. Pre-Session Survey Results: Question 11 108

10. Pre-Session Survey Results: Question 16 109

11. Pre-Session Survey Results: Question 21 110

12. Pre-Session Survey Results: Question 26 111

13. Post-session survey average rankings 112

14. Post- session surbvey: Comparison of student confidence working with LGBT population 113 


\section{INTRODUCTION}

The Association of American Medical Colleges defines cultural competency as: "a set of congruent behaviors, knowledge, attitudes, and policies that come together in a system, organization, or among professionals that enables effective work in cross-cultural situations" (AAMC, 2005).

Cultural competency encompasses a variety of demographics that extend far beyond racial and gender issues; however, not much emphasis is placed on demographics other than racial and gender groups (Hawala-Druy and Hill, 2012; Kelly et al., 2011). In fact, cultural competency is so diverse that some refer to it using different terminology, such as patient centeredness, cultural awareness, and intercultural sensitivity (Beach et al., 2006; Huckabee and Matkin, 2012; Beagan, 2003). Cultural competency forces an individual to examine their own personal views on varying genders, races, socioeconomic status, and sexual preference. The ultimate goal of cultural competence is to gain a level of respect for various cultures, to gain knowledge on varying cultures, and to put aside prejudice and preconceived stereotypes. 


\section{WHAT IS CULTURAL COMPETENCY?}

Just as there are varying definitions of cultural competence, there are varying definitions of culture as well. Lynch and Hanson define culture as the framework that guides and bounds life practices (Lynch and Hanson, 1992). In 2002, Leavitt stated that, culture is not an innate trait from birth, but instead is a learned trait. In the broadest of senses culture can be seen as the physical characteristics, beliefs, attributes, and language that a group identifies as their own (Long, 2011; Simon et al., 2010). Culture depends on both material and non-material aspects (Durand et al., 2012). Culture is dependent on several different factors including age, physical capabilities, gender, migration history, socioeconomic status, and religion. In addition, culture is related to value systems, health beliefs, behaviors, and communication styles (Betancourt et al., 2002; Chapman et al., 2008; Leavitt, 2002; Long 2011; Simon et al., 2010). Having multiple factors influence culture leads to the development of subcultures. Thus, people have many different subcultures which are smaller, distinct units within a larger culture (Leavitt, 2002). Subcultures include more commonly recognized, racial and ethnic groups but also incorporate homosexual and transgender groups which influence the larger study of culture (Long, 2011). An understanding of an individual's main culture and subculture is important in 
understanding the factors that affect a person's preference and use of medical care (Long, 2011). This is the foundation of culturally competent healthcare. Before one can begin to evaluate how cultural competency is taught, a working definition for cultural competency must first be determined.

The concepts of culture and cultural competency have different meanings (Chapman et al., 2008; Leavitt 2002). Leavitt defines a culturally competent healthcare provider as one that acknowledges the immense influence of culture, assesses cross-cultural relations and is vigilant about these cultural differences, expands their cultural knowledge and utilizes their cultural knowledge into everyday practice, and one that adapts to diversity (Leavitt, 2002).

According to the U.S. Department of Health and Human Services' Office of Minority Health, cultural competency is defined as "a set of congruent behaviors, attitudes, and policies that come together in a system, agency, or among professionals that enables effective work in cross-cultural situations," very similar to the definition as the American Association of Medical Colleges $(\mathrm{OMH}$, 2012). Other researchers took the definition of cultural competency one step further by stating that the healthcare provided must "take into account the linguistic and cultural needs" of the patient (Brach et al., 2000; Beach et al., 2006; Leavitt, 2002; Long, 2011; Saha et al., 2008). Larry Purnell affirmed that cultural competence in today's borderless societies is not a luxury, it is a necessity (Clark et al., 2000). Balint (1969) expressed the belief that each patient "has to be understood as a unique human-being" (Saha et al., 2008). Cultural competence in the healthcare arena involves a plethora of areas and concerns. 
Thus, it is a complicated area of research that needs greater attention to develop culturally competent healthcare providers. Consequently, a combination of these similar, but varying, viewpoints is now the standard in teaching cultural competency to today's healthcare professionals. 


\section{HISTORY OF CULTURAL COMPETENCY}

Cultural competency was not addressed in the literature until the late 1980's and early 1990's (Beach et al., 2006; Saha et al., 2008). Early principles that tried to define cultural competence were said to deal with all populations, regardless of background. However, their philosophy focused mostly on immigrant or refugee populations with limited English proficiency and restricted/limited exposure to Western cultural norms (Beach et al., 2006). We now know that cultural competency stretches far beyond those narrow parameters. Since then, various frameworks have been developed, but it was safe to say that it was impractical to predict every situation that a healthcare professional might encounter (Beach et al., 2006). Berlin and Fowkers, Kleinman and colleagues, and Leininger were pioneers in cross-cultural training (Beach et al., 2006; Berlin et al., 1983; Kleinman et al., 1978; Leininger, 1978). They were the first to change the focus from a patient's viewpoint being one part of an underlying disease and shifting it to an understanding of patient's beliefs was necessary in the treatment plan of an underlying disease (Beach et al., 2006). In today's practice, healthcare providers are expected to meet the needs of the patient in the context of the patient's family, community, and cultural setting (Leavitt, 2002). 


\section{THE ROLE OF CULTURAL COMPETENCY IN HEALTHCARE}

Early approaches for cross-cultural training placed the healthcare professional in an environment quite different than their own safe Western civilized society and caused them to look past their own beliefs and find common ground with the patient (Beach et al., 2006). In the late 1980's to the 1990's the scope of what encompassed cultural competence begin to expand in three primary ways. First, cultural competence began to include people of more demographics including those of all color affected by racial disparities. Secondly, cultural competence began to spread to include: prejudice, social determinants, and stereotyping along with their impact on health. Thirdly, cultural competence expanded from interpersonal domain to health care systems and communities (Beach et al., 2006).

One of the major events that occurred to promote increased cultural competence in healthcare was a report published in 1985 by the Department of Health and Human Services Secretary on black and minority health that stated great disparities in the healthcare of people of color (Beach et al., 2006). This report enacted a push to stop healthcare disparities that was highly publicized in President Clinton's administration and gave national attention to the issue of cultural competence (Beach et al., 2006). 


\section{ROLE OF CULTURAL COMPETENCY IN MEDICAL EDUCATION}

Following the political invigoration seen in President Clinton's tenure, studies were launched to find the best approach to teach cultural competency to healthcare providers. In 1989, Cross identified six stages to becoming culturally competent (Cross et al., 1989). These six stages include cultural destructiveness (stage 1), cultural incapacity (stage 2), cultural blindness (stage 3), cultural precompetence (stage 4), cultural competence (stage 5), and the last stage is cultural proficiency (stage 6) (Cross et al, 1989). Campinha-Bacote suggests there are five factors that contribute to culturally competent care (CampinhaBacote, 1991; Leavitt, 2002). The five factors are cultural awareness, cultural knowledge, cultural skill, cultural encounters, and cultural desire (CampinhaBacote, 1991). Cross et al. and Campinha-Bacote's models on cultural competence have been used as a framework for the development of cultural competency curriculum. Amongst the studies, are similar themes that can be found in the plethora of theories that have been developed. A culturally competent healthcare provider healthcare provider is one that (1): adapts to diversity, (2): can look past their own belief system to see the healthcare needs of the patient, regardless if the patient participates in those that conflict with their 
belief system, and (3): can obtain a diverse amount of cross-cultural knowledge that can be used to prevent discrimination of the patient and to provide a welcoming healthcare environment (Beach et al., 2006; Leavitt, 2002).

There has also been a discussion to decide who is responsible for educating health care professionals about providing culturally competent care. Dr. Robert Bolan, M.D., Medical Director of Lambda Medical Group (a health facility that exclusively serves the Lesbian, Gay, Bisexual, and Transgendered (LGBT) community and their families) states that the responsibility should be accepted by both medical schools and residency programs (Butler, 2010). In addition, the American Medical Association's Council on Scientific Affairs, believes that delivering culturally competent care begins in medical school (Butler, 2010).

It is important to note that when exploring cultural competency there is the risk of causing some harm while trying to promote equality. An effort to develop cultural competence programs when related to any particular culture or demographic has the potential to inadvertently lead to stereotyping or racism (Sargent et al., 2005). Lumping one demographic group into a single category or associating every member of a sexual preference with a disease are both examples of the adverse effects of cultural competency training. When providers fail to take sociocultural factors into account they may resort to stereotyping, which can affect their behavior and clinical decision-making (Betancourt et al., 2002). While the teaching of cultural competency has its risks, the benefits of developing and implementing techniques to teach cultural competency to 
healthcare providers far outweighs the risk associated with it. When used effectively, cultural competency training can be of great benefit to the patient and the provider (Huckabee and Matkin, 2012; Beach et al., 2006; Leavitt, 2002;

Betancourt et al., 2002). 


\section{CULTURAL COMPETENCY'S INTEGRATION AT VARIOUS MEDICAL INSTITUTIONS}

Due to the significant impact that cultural competence can have on the quality of care for patients, it is no surprise that it has become an important component in the curriculum in healthcare professions. In 1999, only $8 \%$ of U.S. medical schools taught cultural competence as a part of their regular curriculum (Flores et al., 2000). In 2000, the Liaison Committee on Medical Education released the following standard for medical education:

"The faculty and students must demonstrate an understanding of the manner in which people of diverse cultures and belief systems perceive health and illness and respond to various symptoms, diseases, and treatments. Medical students should learn to recognize and appropriately address gender and cultural biases in health care delivery, while considering first the health of the patient" (AAMC, 2005).

Now fueled by both the Liaison Committee on Medical Education and the Association of American Medical Colleges, approximately $90 \%$ of U.S. medical schools incorporate cultural competence into their curriculum (Boutin-Foster, 2008). There are a variety of traditional approaches used to teach cultural 
competence: Case-studies, didactic, group learning, problem-based, and clerkships, which account for the smallest percentage (Flores et al., 2000; Kripalani, 2006).

Despite the various studies that have shown the importance of training healthcare physicians in cultural competence, $61 \%$ of schools only teach cultural competence in the first two years of medical school (Flores et al., 2000). Even more surprising is the uniformity in the methods used to teach cultural competence, with $87 \%$ of medical schools that incorporate cultural competency in their curriculum using lecture format (Flores et al., 2000). In a recent study found in the Journal of the American Medical Association, the median number of hours spent in medical education was only 5 (Obedin-Maliver et al., 2011; Parkhill et al., 2011). 


\section{CULTURAL COMPETENCY AND THE LGBT COMMUNITY}

Most of the published research on cultural competency deficits involves examining the plight of minorities and women (Leavitt, 2002). Both seem to be logical choices to explore due to the fact that the population of minorities is growing rapidly and women outnumber men. However, one of the most rapidly growing areas of studying cultural competence involves the healthcare of LGBT. According to the president, Joe Solmonese, of the Human Rights Campaign for LGBT Americans, there is now under way a sea of change in the healthcare landscape (McKay, 2011). Healthy People 2020, a program developed by the United States Department of Health and Human Services for health promotion and disease prevention, put forth a new objective to improve the health, safety, and well-being of LGBT individuals (Healthy People, 2020). Stigmatization, political obstacles, prejudice, and methodological challenges all contribute to the limited populations that are available for research studies (Barker, 2012). Despite all of the leaps and bounds being made to improve the overall quality of healthcare for the LGBT community, the National Institutes of Health $(\mathrm{NIH})$ does not categorize the LGBT community as a minority population for research purposes when evaluating health disparities amongst minorities (Mollon and Stahl, 2010). In the U.S., accurate population data is not available for the LGBT 
community because questions related to sexual preference are rarely included in national health surveys (Baker et al, 2009). Due to the lack of funding, research done on health disparities amongst the LGBT community involves a much smaller scale of subjects and limited geographic area (Mollon and Stahl, 2010). The LGBT community itself is united by suffering from the same prominent issue, stigma, there is still more research needed to look at the cross cultural issues. The LGBT community encompasses a vast range of racial demographics, socioeconomic status, and educational backgrounds. To change the disparity experienced by many of these patients, research is needed to not only look at the negative effects that their sexual orientation has in their healthcare needs. Additionally, cross-cultural analysis of the other factors outside sexual orientation may affect their healthcare needs is necessary. An example of this approach would be to examine the healthcare concerns of a minority lesbian woman. An examination of her healthcare needs as a woman, a minority, and a member of the LGBT community would all need to evaluated.

Some may ask, "Why is it a concern for me?". The answer to that question is that neglecting to address the specific issues of a particular community, while ethically wrong, also leads to the following medical disparities for that community. These disparities could include an increase in transmission and progression of diseases, a decrease in mental and physical well-being, an increase in health costs, and a decrease in longevity (Healthy People 2020).

Studies have shown that members of the LGBT community limit visits to see healthcare providers due to fear of discrimination, previous encounters with 
homophobic attitudes from healthcare providers, and stress due to negative social perception (Mollon and Stahl, 2010). Prejudices like these can make it very difficult for a LGBT patient to disclose their sexual identity to their healthcare provider (Coren et al., 2011). If an LGBT patient perceives a negative attitude toward the LGBT community, it can induce a fear of disclosing sexual identity information and this information is very important due to their unique healthcare needs (Healthy People 2020). A 2001 Kaiser Family Foundation (KFF) survey revealed that only about $64 \%$ of gay and lesbian respondents had voluntarily told their doctors about their sexual orientation (KFF, 2001). Roughly $36 \%$ of respondents still felt uneasy about relaying their sexual preference (KFF, 2001).

Horrific stories have been widespread of cases in which healthcare providers failed to provide a safe place for patients. Two senior lesbian women were thrown out of a healthcare facility by the clinical director and called "faggots" (Mollon and Stahl, 2010) Another story tells of a young woman who went for a check-up due to an unfavorable cough and left with a handful of unrequested Bible scriptures unrequested by her (Mollon and Stahl, 2010). LGBT patients have identified the following issues during physician visits: heterogeneity of patient registration forms, heterogeneity of patient satisfaction survey, negative clinical interactions, and lack of tracking healthcare outcomes.

Due to these four major concerns, many LGBT patients seek alternative healthcare options at specialized environments of LGBT community health centers (Bruessow, 2011). However, these options are not available globally and many LGBT patients choose to forfeit appropriate healthcare as a result. 
Approximately twice as many lesbian females, as compared to heterosexual females, delay obtaining healthcare due to past negative experiences (Mollon and Stahl, 2010). As a healthcare provider, it is one's responsibility to eliminate possibilities for humiliation while a LGBT patient is seeking care. According to Lambda Legal, a national organization advocating for LGBT equality, "disclosure of sexual orientation can be a Catch-22" (McKay 2011). This is a devastating, but quite true reality for many members of the LGBT community. Disclosure of sexual orientation can lead to discrimination; however, lack of disclosure can lead to inadequate healthcare. Lambda Legal put this theory to the test and conducted a survey in 2009 along with 100 partner organizations (Lambda Legal,2009; McKay, 2011). The survey had 4,916 respondents consisting of LGBT patients and people living with HIV showed that a shocking $8 \%$ of LGBT patients had been outright denied healthcare and $10 \%$ had harsh language used towards them (Lamda Legal,2009; McKay, 2011). These previous studies and surveys highlight why members of the LGBT communities fear disclosing their sexual history due to fear of judgment by healthcare workers.

Because of inadequate assessments and preventative services from providers, as well as a higher prevalence of smoking and obesity, gay men and lesbian women have an increased risk of lung, cervical, breast, and anal cancer (Mollon and Stahl, 2010). Additionally, LGBT youth are 2 to 3 times more likely to commit suicide (Healthy People 2020). Further evidence of the lack of preventative services is observed with gay and bisexual men accounting for $53 \%$ of new HIV cases (Mollon et al., 2010). Other health concerns include the high 
rates of obesity among lesbian and bisexual women that are overweight and obese, high rates of tobacco, alcohol, and other drug use in the LGBT population, and a lack of social services for elderly LGBT patients (Healthy People 2020). A link between discrimination of LGBT patients and psychiatric disorders, substance abuse, and suicide has been established (Healthy People 2020).

Many healthcare providers do not have any negative feelings toward the LGBT community. However, even physicians with the best of intentions can project inaccurate perceptions that they are not sensitive to LGBT issues. By making minor, but effective, changes one can provide a healthcare environment that conveys to LGBT patients that they are with: (1): a knowledgeable staff aware of LGBT healthcare issues, (2): healthcare providers that are sensitive to their diverse needs and identities, and (3): a healthcare staff that is able and willing to make interpersonal and office-related adjustments to provide them with the best care (Coren et al., 2011).

Breaking down barriers that cause an unpleasant experience for LGBT patients is essential. A first step that many physicians need to make is evaluating one's own belief system (Coren et al., 2011). If there is any discomfort when treating a LGBT patient, a physician can seek help from organizations that promote LGBT affirmative practice such as the American Medical Association's Gay, Lesbian, Bisexual, and Transgendered Advisory Board (Coren et al., 2011.) 
Beyond the conceptual components of evaluating belief systems and attitudes, there are specific actions that healthcare providers can perform to provide a safe environment for their patients. Specific actions should include adjusting the patient intake form and creating an atmosphere of acceptance in the office setting (Mollon and Stahl, 2010). The exact method of teaching healthcare providers how to address those barriers is still being researched. 


\section{MODES OF TEACHING CULTURAL COMPETENCE TO HEALTH SCIENCE STUDENTS}

Cultural competency has been taught as a part of health science students' curriculum for many years now due to an increase in diversity and mandates set on a national level (AAMC, 2005; Boutin-Foster, 2008; Durand et al., 2012; Flores et al., 2000; Kripalani et al., 2006). The overall goal of cultural competency training is to equip future providers with the knowledge and skills to have a full understanding of cultural differences in order to provide an effective clinical experience for patients (Long, 2011). There are a variety of models used to teach cultural competency including the Cross Model on Cultural Competence, Purnell Model on Cultural Competence, The Campinha-Bacote Model on Cultural Competence, and many others (Hoban and Ward, 2003; Cross, 1989; Leavitt, 2002; Purnell, 2002; Campinha-Bacote, 1992). The principles or steps in the varying models differ, however many of the teaching models involve one of three conceptual approaches for teaching cultural competence. The three conceptual approaches to teaching cultural competence are knowledge-based, attitudebased, and skill-building based (Kripalani et al., 2006). Knowledge -based concepts include working through the definitions of cultural competency and different concepts involved in cultural competency (Kripalani et al., 2006). 
Attitude-based concepts on cultural sensitivity and cultural awareness (Kripalani et al., 2006). Lastly, in the skill-building approach educators focus on communication skills and the patient-physician relationship (Kripalani et al., 2006).

Traditionally, incorporation of these three approaches is seen throughout many health science cultural competency training formats. Training takes place in the form of lectures, clerkships, group discussions, standardized patients, language training, elective courses, and workshops (Crandall et al., 2003; Kripalani et al., 2006; Long, 2011).

Cultural competency training is rarely seen as a separate course; most often cultural competence is taught through a case-study, intermittent lecture, or a workshop (Kripalani et al., 2006). Frequently, cultural competency is integrated across the curriculum rather than seen as a standalone course (Crandall et al., 2003; Beamon, 2006; Long, 2011). In fact, in 2000 it was reported that only $8 \%$ of medical schools offered cultural competence as a separate course (Beamon, 2006; Flores et al., 2000). The schools that do offer some form of cultural competence training usually provide it through some form of clinical internship. Many schools immerse students in a clinical experience in an isolated, diverse, unfamiliar community (Beamon, 2006). The goal of such an experience is to introduce students to diverse communities with real-life experience working with minority populations. 


\section{POTENTIAL OF VIRTUAL WORLDS FOR CULTURAL COMPETENCY INSTRUCTION}

Newer approaches to cultural competence training involve the use of virtual worlds. Virtual worlds are online environments were individuals are capable to communicate and interact in a realistic manner (Bainbridge, 2007). There are many benefits to using virtual worlds for cultural competency training including: anytime learning, ability to change language and content, consistency in training, and the ability to practice in a safe, non-judgmental environment (Bauman, 2012; Richardson et al., 2013; Rutledge et al., 2008). Virtual worlds allow for students to put on various identities and reflect on the consequences of their actions (Games and Bauman, 2011). Virtual worlds have been shown to increase student engagement and learning abilities of students (Annang et al., 2010; Giddens et al., 2011; Hussainy et al., 2012; Twining, 2009). The River City Project is an online virtual world funded by the National Science Foundation that uses a virtual city, known as River City, to teach middle-school students about rising health problems (Annang et al., 2010). A second example is Whyville, a virtual world for pre-teens and teens to learn about a plethora of subjects including science, civics, public health, and economics (Annang et al., 2010). 
There are several reasons for the growing popularity of virtual worlds in education. The growth of virtual worlds in education can be likened to that of the growth of entertainment in education (Annang et al., 2010; Singhal and Rogers, 1999). Entire networks over the years have been dedicated to educating students and now entire websites are dedicated to educating students. Just as using entertainment to educate students has its benefits, the use of virtual worlds in education, has its benefits as well. The greatest of these benefits is anonymity (Annang et al.; Baker et al., 2009; Hansen, 2008; Richardson et al., 2011; Wiecha et al., 2010). Through virtual worlds students are allowed to freely engage in group discussion without scrutiny or wayward looks (Sweigart and Hodson-Carlton, 2013; Weicha, 2010). Virtual worlds have been shown to promote collaboration and self-help in areas of drug abuse, sexual health, and health promotion (Annang et al., 2010).

Virtual worlds allow the student to learn in a diverse and unique cultural experience. Virtual worlds provide a venue to custom tailor vignettes to the diverse population of study (Annang et al., 2010; Giddens et al., 2012; Rutledge et al. 2008). Unlike real-world scenarios, virtual worlds have the potential to introduce students to a diverse population in a short-period of time (Bauman, 2012). In fact, in 2009, it was estimated that there were over 300 million registered users in virtual worlds (Hays, 2008; Warburton, 2009). These varying demographics allow for a single case-study to involve a different ethnic minority, gender group, age demographic, or sexually diverse patient all in one scenario. One of the most rapidly growing populations in the United States is the LGBT 
community and immediate and ongoing research studies are needed to assist with disparities seen in their healthcare. In a constantly growing and everevolving society a teaching tool that can change as quickly as the world does is essential and for the LGBT and other communities, the use of virtual worlds may serve as an effective teaching pedagogy.

Some studies have previously explored the teaching of cultural content using a virtual world. In one study, Rutledge provided a student virtual clinical experience with virtual patients. Students logged into a virtual hospital and had the ability to perform an in-world real patient history report. Students were provided feedback through previously programmed responses from the patients (Giddens et al., 2012; Rutledge et al. 2008). Results of this study showed that virtual worlds are a useful teaching tool for teaching students how to deal with culturally diverse population when taking patient health history reports. 


\section{SECOND LIFE ${ }^{\text {TM }}$ AS A POTENTIAL TOOL FOR CULTURAL COMPETENCY TRAINING}

While several different virtual worlds exist today, such as the River City Project and Whyville, have been utilized for medical education, one could argue that Second Life ${ }^{\mathrm{TM}}$ is the most popular virtual world in today's era and the most frequently used 3-D world in education.

Launched by Linden Lab on June 23,2003 , Second Life ${ }^{\text {TM }}$ is a 3-D virtual community in which individuals can communicate and interact with each other using avatars. Second Life ${ }^{\mathrm{TM}}$ has over six million virtual residents and offers a broad range of possibilities for the educational community. Second Life ${ }^{\mathrm{TM}}$ has the distinction of housing land used for teaching courses and research for over three hundred universities and colleges, (Weicha, 2010; Richardson et al., 2011). Many universities use Second Life ${ }^{\mathrm{TM}}$ to create a replica of portions of their actual campuses which serves as great tool for recruitment (Boulos et al., 2007; Richardson et al., 2011).

The literature contains publications on the vast array of uses of Second Life $^{\mathrm{TM}}$ are vast and educational potential is limitless. Ohio University offers an island where students can learn about nutrition. San Jose State University 
provides cardiac auscultation training for their clinical students, and the University of California has an island on Second Life ${ }^{\mathrm{TM}}$ that educates people on Schizophrenic hallucinations (Boulos et al., 2007; Richardson et al., 2011).

Other basic science disciplines use Second Life ${ }^{\mathrm{TM}}$ as a teaching tool to engage students. The Gene Pool in Second Life ${ }^{\mathrm{TM}}$ is an island dedicated to teaching students on genetics. Students can visit the island and learn about various topics in genetics and even explore a 3-D eukaryotic cell (Boulos et al., 2007). In addition, the University of Kentucky teaches an online virtual anatomy course in Second Life ${ }^{\mathrm{TM}}$ in which students can take part in video-guided tutorials and walk through a 3-D pteyrgopalatine fossa (Richardson et.al, 2011).

The use of Second Life ${ }^{\text {TM }}$ for educational purposes is not just limited to colleges and universities. National organizations also have begun to use Second Life $^{\mathrm{TM}}$ for educational purposes for the public. HealthInfo Island is sponsored by the U.S. National Library of Medicine and is a tool for consumers to find health information (Gorini et al., 2008). Other National organizations that make use of simulation within Second Life ${ }^{\mathrm{TM}}$ are the U.S. Centers for Disease Control and Prevention, U.S. National Oceanic and Atmospheric Administration, the Virginia Tech Memorial, the Van Gogh Virtual Museum, and the American Cancer Society (Jarmon, 2008; Beard et al., 2009).

One of the newest areas of study involving Second Life ${ }^{\mathrm{TM}}$ is its use in anatomical science. The University of Kentucky College of Medicine constructed a virtual anatomy lab for undergraduate and health professional students 
(Richardson et al., 2011). Students can participate in interactive lessons that give background on anatomical structures, provide in-world quizzes, and delivers additional resources (Richardson et al., 2011).

There are multiple benefits in using Second Life ${ }^{\mathrm{TM}}$ in an educational setting. The first of these is the ability for both students and teachers to customize their own unique avatar. Some studies have shown that students tend to gravitate to an avatar that resembles their own ethnic background (Boulos et al., 2007). Some suggest this may lead to a more comfortable learning environment as opposed to traditional learning environments because students choose to learn from avatars that reflect their own real life appearances (Boulos et al., 2007).

Second Life ${ }^{\mathrm{TM}}$ also provides an easy to navigate experience for students compared to a traditional classroom setting, in which all students potentially can have a clear, great view (Boulos et al., 2007; Richardson et al, 2011). Students can walk, run, and even fly around the learning environments (Boulos et al., 2007; Richardson et al., 2011). This type of environment allows students to engage in trial and error type practice without risks of serious repercussions (Boulos et al., 2007; Hewitt et al., 2008; Foss, 2009; Richardson et al., 2011). Also, studies have shown that virtual learning environments facilitate reflective practices (Boulos et al., 2007). Students are more likely to discuss and share information learned from virtual learning centers compared to traditional classroom settings. Lastly, e-learning environments can provide a significant cost savings compared to traditional instructor-led classrooms (Ruiz et al., 2006). One 
can conclude this is due to savings from instructor training costs, labor costs, and travel costs (Kripalani et al., 2006; Ruiz et al., 2006). Also, there is a reduction in the costs of educational publications (Ruiz et al., 2006).

Despite the numerous benefits of Second Life ${ }^{\mathrm{TM}}$, there are some challenges. The challenges and caveats that come with the use of Second Life ${ }^{\mathrm{TM}}$ are not much different from those associated with other social networks such as MySpace and Facebook. Internet addiction, gambling, violence, pornography, privacy issues, and vandalism are just some of the negative aspects of Second Life $^{\mathrm{TM}}$ (Boulos et al., 2007). Also, there may be some accessibility issues for disabled and elderly members of the community (Boulos et al., 2007). Due to virtual worlds requiring adequate dexterity skills, some members of the community may find this type of learning environment more of a challenge than a benefit. Despite the challenges associated with Second Life ${ }^{\mathrm{TM}}$, it still has the potential to be a useful teaching pedagogy in the teaching of culture competence. 


\section{CULTURAL COMPETENCY WORKSHOPS AT THE UNIVERSITY OF LOUISVILLE SCHOOL OF MEDICINE}

The University of Louisville's Cultural Competency workshop began in 2006. During the workshop, students, faculty, and staff attend a half day workshop centered on diversity in healthcare. The 2010 workshop hosted over 400 individuals from a variety of health care fields including medicine, dentistry, and audiology. Breakout sessions were offered on specific topics related to diversity in the medical field, including Japanese Acupuncture in Western Society, Mennonite Culture and Health-Care Provisions, Working Effectively with LGBT Patients, Aspects of Practical Difference between Islamic and Western Medicine, Applying Military Cultural Competency to the Care of Veterans/Service Members, Child Discipline \& Maltreatment: How Do We Define Abuse \&Neglect in a Multicultural Society, and Students \& Their Viewpoints on Culture.

Recognizing the need to integrate LGBT cultural competency into the health science program, the University of Louisville School Of Medicine set out to provide a workshop that provided health science students and professionals an opportunity to strengthen their knowledge of the LGBT community and aid them in providing better care for their patients. The Working Effectively with LGBT Patients Workshop was a workshop in the series that in the past had low student 
buy-in and poor student interest. The University wanted to branch out from the traditional methods of investigating the importance of cultural competence and integrate innovative ways to engage students and increase student interest in the LGBT workshop session.

In the present study we set out to look at Second Life ${ }^{\mathrm{TM}}$ as a tool to enhance the Working Effectively with LGBT Patients session of the Cultural Competency workshop. Our hypothesis was that the Second Life ${ }^{T M}$ LGBT workshop setting will enhance student interest and mastery of the sensitive nature of LGBT disparities in the healthcare industry compared to the traditional workshop setting. We had a specific aim of determining if Second Life ${ }^{\text {TM }}$ is a more effective form of introducing LGBT issues to first year health science students as compared to the traditional workshop setting. 


\section{METHODS}

\section{UNIVERSITY OF LOUISVILLE CULTURAL COMPETENCY WORKSHOP SERIES}

Starting in 2006, the University Of Louisville School of Medicine developed a series of workshops to address issues in diversity as it relates to the healthcare industry. The workshops are designed for health science professional students, residents, and faculty. Each year the half-day workshop series works under the theme of "Increasing Cultural Competency in Our Changing World". Breakout sessions are offered in various topics on diversity that are currently important to the healthcare community. Volunteer facilitators aid the students through the information and activities relevant to each workshop topic. This workshop series is organized by the Office of Diversity and Inclusion and is offered to students at no cost.

On September 27, 2012 the seventh annual University of Louisville Cultural Competency Workshop Series took place on the Health Sciences campus of the University of Louisville. A total of 343 participants attended the workshop series, consisting of students from the schools of Audiology, Dentistry, 
and Medicine. The students came from a variety of ethnic groups: African American, American Indian, Asian, Caucasian, Hispanic, and Mixed Race. The 343 participants completed a pre-session anonymous questionnaire consisting of questions related to each of the different break-out sessions held during the conference. The program for the 2012 conference, outlining the topic areas addressed in each breakout session is included in FIGURE 1.

Our study focused specifically on the LGBT content associated with this conference, addressing the results of the pre-conference questionnaire on LGBT cultural competence, and the efficacy of the two LGBT break-out sessions. 


\section{PRE-QUESTIONNAIRE SURVEY}

The pre-questionnaire survey questions were compiled from The

Providers Guide to Quality and Culture Quiz@ (http://erc/msh.org/mainpage.cfm). The pre-session questionnaire consisted of twenty-six questions, of which six of the questions, assessed students' attitudes about the LGBT community before attending any of the Cultural Competency sessions. Students were asked to indicate if they strongly agreed, agreed, were uncertain, disagreed, or strongly disagreed to each of the statements. The questions on the pre-session survey were:

- Question 5: Access to health care is the same for LGBT persons as for other members of the population.

- Question 8: Most LGBT young people do their first "coming out" to a nonparent adult (e.g. a teacher or doctor).

- Question 11: LGBT people are less likely than heterosexual people to be in long-term monogamous relationships.

- Question 16: LGBT patients have a unique set of health risks and health needs. 
- Question 21: As a healthcare provider I feel it is important for me to know about my patient's sexual orientation, sexual practices, and gender identity.

- Question 26: I would prefer not to treat patients with gender issues. 


\section{ORGANIZATION OF THE LGBT BREAK-OUT SESSIONS}

After attending the preliminary session and completing the pre-session questionnaire, students were released to their break-out sessions, which included Japanese Acupuncture in Western Society, Mennonite Culture and Health-Care Provisions, Working Effectively with LGBT Patients, Aspects of Practical Difference between Islamic and Western Medicine, Applying Military Cultural Competency to the Care of Veterans/Service Members, Child Discipline \& Maltreatment: How Do We Define Abuse \&Neglect in a Multicultural Society, and Students \& Their Viewpoints on Culture (FIGURE1).

Two separate LGBT sessions were held, a traditional lecture type of session that was used in previous years and a workshop that involved the use of Second Life ${ }^{\mathrm{TM}}$ as a component of the workshop. Our study had an experimental group of twelve students who participated in the Second Life ${ }^{\mathrm{TM}}$ enhanced workshop setting and a control group of forty-two students who participated in the traditional lecture workshop setting. The small study size was implemented for the Second Life ${ }^{\mathrm{TM}}$ enhanced session due to it being a pilot study. Students were randomly selected from the pool of students enrolled in the Working Effectively with LGBT Patients session and placed in either the Second Life ${ }^{\mathrm{TM}}$ enhanced or traditional workshop session. Both the Second Life ${ }^{\mathrm{TM}}$ group and the control group participated in the pre-questionnaire survey administered to all participants in the 
workshop series, as well as a LGBT post-session survey. All components of the workshops were the same in Second Life ${ }^{\mathrm{TM}}$ workshop and the traditional workshop, with the exception of the administration of the scenarios.

Both LGBT sessions were one hour long and consisted of the same four components: Blanket Exercise, Scenario, Reflection, and Ways of Finding resources/Ongoing Education. As a way of setting the stage for dialogue, presenters facilitated an experiential blanket exercise with the goal of both highlighting the barriers LGBT people face when accessing quality health care, as well as creating a level of discomfort among participants with the goal of increasing empathy for patients. In this exercise a student volunteer was asked to come to the front of the classroom and the student audience was asked to identify various barriers that the LGBT communities face. As each barrier was identified, a blanket was placed over the students head as a symbolization of the weight that the LGBT community carries on a daily basis. The student volunteer and the student audience were asked to reflect on how this exercise helped them to relate to the plight of the LGBT population. At the conclusion of the exercise, participants were invited to state ways in which they could be allies to LGBT patients and create a more positive health care experience. Participants were then placed into small groups to discuss scenarios, focused on LGBT patient experiences. Each group considered a series of questions related to their assigned scenario and presented their findings to the larger group. As facilitators debriefed the exercise, they invited feedback from other participants, shared 
terms and definitions, and provided data regarding risk factors associated with each scenario. The session concluded with an open Q\&A session.

In both the Traditional and Second Life ${ }^{\text {TM }}$ LGBT sessions, the same two scenarios were used for each session; however, the two workshops differed in the manner in which the scenarios were delivered to the students. In the Second Life $^{\mathrm{TM}}$ workshop setting the scenarios were transformed into scripts to be played out in-world for the attendees of the Second Life ${ }^{\mathrm{TM}}$ session to view. Also, in the Second Life ${ }^{\mathrm{TM}}$ workshop setting students were able to see the scenarios played out on the classroom screen, as it was being reenacted in the Second Life ${ }^{\mathrm{TM}}$ virtual world. The scenarios were projected on the classroom screen and the students were able to see the action, much like if they were watching a movie. In the traditional workshop setting the case scenarios were read from a sheet of paper and the students were asked to give feedback on each scenario they heard; the format that was used in previous years. The traditional workshop session did not have the scenarios in script form but, simply in a short-story paragraph form. Both groups had a short introductory synopsis of the scenario that was read out loud to each session before the scenarios were either read or enacted out for the Second Life ${ }^{\mathrm{TM}}$ session. 


\section{PREPARATION OF THE SECOND LIFE ${ }^{\mathrm{TM}}$ WORKSHOP SESSION}

To prepare for the Second Life ${ }^{\mathrm{TM}}$ session a hospital and a doctor's office design plan was created and built by a professional Second Life ${ }^{\mathrm{TM}}$ builder, Matt

Hazzard, Medical Illustrator in the Academic Technology group at the University of Kentucky (FIGURE 2, FIGURE 3). Mr. Hazzard was given a design plan that was developed through online research, visiting of actual healthcare facilities, and consultation with healthcare providers to create an authentic in-world experience.

A script based on the scenarios that were to be used in the Traditional lecture workshop was developed. No details of the action were changed, but they had to be delivered to the audience by both dialogue and gestures from the avatar. For example, when the traditional group was reading, "the patient scanned the office...," the Second Life ${ }^{\mathrm{TM}}$ group actually saw the patient/avatar scanning the office through their body movement and not their suggestions. After several revisions, and mock run-throughs, volunteers from the University of Kentucky and University of Louisville were given the scripts and additional inworld practices took place to create an authentic experience for the students. In the first scenario, there were three volunteers that had speaking roles and three volunteers that acted as patients waiting in the waiting room of the doctor's office to make it a more authentic experience. In the emergency room scenario, 
scenario 2, there were three volunteers who had speaking roles and four volunteers that had supporting, background avatar roles.

In addition to creating the in-world environments and developing a script for the avatars, preparations were made to equip each Second Life ${ }^{\mathrm{TM}}$ actor/actress for the live performance at the Second Life ${ }^{\text {TM }}$ LGBT break out session. Each computer that was to be operated by a volunteer to control an avatar had to register with Second Life ${ }^{\mathrm{TM}}$ and download and install the Second Life $^{T M}$ Viewer. A step-by-step guide was provided to each volunteer to ensure that this task was accomplished as smoothly as possible (FIGURE 4). Volunteers were encouraged to take several weeks to learn the Second Life ${ }^{\mathrm{TM}}$ technology and practice navigation skills in-world. The last step in equipping the volunteers was to provide them with Logitech ${ }^{\mathrm{TM}}$ microphones and headsets so that they could hear and participate in the dialogue that the student audience would experience (FIGURE 5). 


\section{THE SCENARIOS}

\section{Scenario One:}

The first LGBT breakout session scenario presented involved LaTonya, a lesbian woman, who visits the doctor's office after a very long time. She enters the doctor's office and is greeted by a receptionist. Upon entering, LaTonya begins to search for any clues that the doctor's office is an LGBT friendly office environment. When called to the examination room, a doctor avatar proceeds to go over the intake form with the patient and fills in some gaps on her intake form. The students were charged with identifying positives and negatives that they observed in the scenario. The script used for scenario one follows.

\section{Scenario One: Office case script}

Facilitator provides the audience with some background:

LaTonya Jones is a 35 year old lesbian woman. She scheduled an appointment with the family medicine clinic because she is required to have a physical exam for work. She has not had a physical in over 10 years because of past experiences with health care professionals who responded negatively to her sexual orientation. 
Please watch the following scenario and be prepared to comment on any positive and negative aspects of the encounter. Also consider the likelihood that LaTonya will return to this office for routine preventative care.

In world:

http://maps.secondlife.com/secondlife/University\%20of\%20Louisville/146/18/24

Avatars: LaTonya (Ashley Mack), Mrs. Flowers (April Richardson-Hatcher), Nurse Floyd (Kendall Gault), Doctor (Matt Hazzard), Patients in waiting room (Christena Gazave, Nicole Herring, Jennifer Brueckner)

LaTonya (Ashley Mack) enters the reception area of the doctor's office, looking around at the office postings. She sees mixed signals regarding whether this office is safe, inclusive, and non judgmental for LGBT patients.

LaTonya (Ashley Mack) goes to the front desk, telling the receptionist Mrs. Flowers (April Richardson-Hatcher): "I scheduled an appointment with your office because I am required to have a physical exam for my new job."

Mrs. Flowers (April Richardson-Hatcher): "Okay, we can definitely help you with that!" in a very friendly tone. "You just need to fill out this intake form. Our HIPPA policy is included with the intake form and our nondiscrimination policy is posted here (point to wall to the right of the reception desk). "

LaTonya (Ashley Mack) reads through the non discrimination policy so that the audience can clearly read over her shoulder.

After LaTonya finishes reading the policy and sits down, the nurse calls her back.

Nurse Floyd (Kendall Gault): "LaTonya? We are ready for you now. You can finish the intake form in the exam room. "

LaTonya (Ashley Mack) follows the nurse into the exam room and sits on the exam table.

LaTonya (Ashley Mack) reads through the intake form. We can see the questions. She focuses in on the social history questions. Sighs and says to herself "I thought this place would be different." 


\section{Patient intake form}

Please fill out the following:

Medical history (check if you have any of these conditions):

- Hypertension

- Thyroid disease

- Depression

- High cholesterol

- Cancer

- Heart disease

- Diabetes

- Anxiety

- Asthma

o Other:

\section{Social history}

Gender:

Male

Female

Marital status: $\quad$ Married Single Widowed Divorced

If married, provide spouse contact information: Name:

Phone:

Number of pregnancies:

Number of children:

Number of abortions/miscarriages:

Occupation:

Religious preference:

Have you ever smoked cigarettes? Yes No

If so, number of years and how often?

Do you smoke now?

Yes No 
Doctor (Matt Hazzard): "Hello Ms. Jones, my name is Dr. Simmons. What brings you in to see me today?"

LaTonya (Ashley Mack): "I just started a new job and my employer is requiring me to get a physical exam. I haven't had one in 10 years."

Doctor (Matt Hazzard): "That is a long time! Why not?"

LaTonya (Ashley Mack): "I've had bad experiences with doctors in the past."

Doctor (Matt Hazzard): "I am sorry to hear that. What happened?"

LaTonya (Ashley Mack): "They made me feel uncomfortable and out of place. It's like they don't listen; just hear what they want or expect to hear."

Doctor (Matt Hazzard): "I want to work with you to prevent that from happening here. Please tell me if I do or say anything that makes you uncomfortable, ok? Ok, to get started, I have some questions for you. You didn't complete part of your intake form, so I need to fill in those answers. Are you married?"

LaTonya (Ashley Mack): (Startled) "Uh (pause), no."

Doctor (Matt Hazzard): "Are you in a relationship? "

LaTonya (Ashley Mack): "Uh, yes."

Doctor (Matt Hazzard): "How long have you two been together?"

LaTonya (Ashley Mack): "About 4 years."

Doctor (Matt Hazzard): "Do you live with him?"

LaTonya (Ashley Mack): "Uhh...actually, my partner is a woman."

Doctor (Matt Hazzard): "Oh! Excuse me for a moment...." And steps out into the hallway."

In the hallway,

Doctor (Matt Hazzard): "Nurse Floyd, would you please finish taking this patient's health history? I think she would be more comfortable talking to you."

Nurse Floyd (Kendall Gault): "Yes doctor." And walks into the exam room.

\section{END OF SECOND LIFE ${ }^{\text {TM }}$ SCENARIO ONE SCRIPT}




\section{Follow-up to Scenario One (Both Second Life ${ }^{\mathrm{TM}}$ and Traditional)}

Facilitator: Based on what you just saw:

Can you identify some positive things about this encounter?

Can you identify some negative aspects of this situation?

Based on this experience, how likely is it that LaTonya will feel safe sharing her personal health history with you?

How likely is it that LaTonya will return to your office for routine preventative care and tell others about her experience?

Positive signs:

Rainbow symbol on bulletin board, LGBT literature on the coffee table

Negative signs:

No gender inclusive restroom (separate male or female restrooms). Many transgender or other LGBT patients not conforming to physical gender stereotypes have been harassed for entering the "wrong" bathroom so at least one restroom without Men or Women labels would create a safer and more comfortable atmosphere.

Nondiscrimination policy does not include sexual orientation

Intake form does not include inclusive choices for LGBT patients: rather than marital status, relationship status, including options like "partnered". Adding a "transgender" option to the male/female checkboxes help you capture better information about transgender patients, and will be an immediate sign of acceptance.

If a patient has left blanks on the intake form, this may be an indication that they felt uncomfortable being open in writing. You have another, better chance to create trust with the patient during the interview.

Avoid the assumption that your patients are heterosexual just because they haven't told you otherwise.

\section{Mute computer screen until next scene is set up.}




\section{Scenario Two:}

A transgendered patient is rushed to the emergency department (ED) after a car accident. Shortly after arrival the ER doctor notices that the patient has not been treated due to the indifferent attitudes of the emergency medical service (EMS) workers. A conversation proceeds between the doctor and the ambulatory workers. Again, students are asked to identify the positives and negatives in the scenario.

\section{ER case script}

Facilitator provides the audience with some background:

You are a physician in the ER. One night, a traffic accident occurs and the victim is rushed to your hospital. When the victim arrives, the doors of the ambulance are opened, and the entire EMS team is standing around the gurney, staring at the victim.

\section{Avatars: Patient (Jennifer Brueckner) ; Doctor 1 (Ashley Mack), Nurse 1 (April Richardson-Hatcher), EMS 1 (Matt Hazzard), EMS 2(Kendall Gault), Doctor 2 (Nicole Herring), Nurse 2 (Christena Gazave)}

Gathered around gurney is Nurse 1, EMS 1, EMS 2, Doctor 2

Nurse 2 is behind the desk at the computer in the ER.

Nurse 1(April Richardson Hatcher): Rushes into the ER from the ambulance, shouting "Doctor, we need you out here!" 
Doctor 1(Ashley Mack): Rushes out to the ambulance to find the crowd of people gasping and whispering around the victim on the gurney.

Doctor 1(Ashley Mack): " Let me through....what is the status of this patient?"

EMS worker 1 (Matt Hazzard): "Hah! Do you really want to know?"

Nurse 1(April Richardson Hatcher): "The patient is a 26 year old, who was traveling at a high rate of speed when she lost control of the car, hitting a utility pole and then a tree. The driver was trapped in the car for around 30 minutes and has an open leg wound that is bleeding significantly."

Doctor 1(Ashley Mack): "Nothing has been done for this patient! help me get her inside.....we need to stabilize her immediately."

EMS worker 1(Matt Hazzard): laughing as they talk; "You might want to put "IT" in a private room, doc."

EMS worker 2 (Kendall Gault): "Yeah, doc, this one's all yours!"

Patient moved into triage area, followed by doctor and 2 nurses. EMS team hangs around and gawks.

\section{Another doctor 2 (Nicole Herring) takes charge of the patient.}

Doctor 1(Ashley Mack): To nurse...." What was that all about? I have worked with those EMS guys for years and never seen them act like this. I'm surprised this victim is still alive; it doesn't look like she was treated at all in the ambulance."

Nurse 1(April Richardson Hatcher): "Apparently, when they arrived on the accident scene and went to examine the victim to assess injuries, they had found that the victim had a penis and refused to treat the patient. They wouldn't touch the patient or even do basic life support."

Doctor 1(Ashley Mack): "I've never seen such negligence. What's the matter with you two?"

EMS worker 1 (Matt Hazzard): "At first, we thought she was a lady but when we saw she was, well whatever, we had never seen that before. I really have a problem with people like that and want nothing to do with them. "

EMS worker 2 (Kendall Gault): "I agree completely. It was just too weird for me and I couldn't bring myself to touch the victim; I didn't want to contract HIV with all of that blood." 
Doctor 1(Ashley Mack): "You should be aware that I will be reporting both of you for negligence and if you ever try something like that again, you'll have a lot more to answer to."

\section{END OF SECOND LIFE ${ }^{\mathrm{TM}}$ SCENARIO ONE}

\section{Follow-up to Scenario Two (Both Second Life ${ }^{\mathrm{TM}}$ and Traditional)}

Facilitator provides the audience prompts audience discussion with the following questions:

- Can you identify the positive aspects of this situation?

- Can you identify the negative aspects of this situation?

- How would you have reacted if you were the doctor in this situation?

- Would you have handled things differently? Why or why not?

The students from both sessions were asked to evaluate the positives and negatives that went on in each scenario and give feedback on the lessons learned through the scenario. 


\section{POST-SESSION QUESTIONNAIRE}

In addition to the pre-session survey that was completed by all conference participants, the students who attended either the Traditional and Second Life ${ }^{\mathrm{TM}}$ LGBT session took a separate post-session questionnaire (FIGURE 6). The postsession survey questions were adopted from the study conducted by Kelley et al. published in their journal article entitled "A Critical Intervention in Lesbian, Gay, Bisexual, and Transgender Health: Knowledge and Attitude Outcomes Among Second-Year Medical Students" (Kelley et al., 2008). There were eleven questions on the post-survey administered to both the Traditional and Second Life $^{\text {TM }}$ LGBT group. Students were to evaluate most questions on a scale from 1 to 5 , with 1 being poor and 5 being excellent. The questions on the post-survey question were:

1. Rank in order the four components of the session from (1) most important to (4) least important, which are, blanket exercise, scenarios, reflection, and discussion of where to find resources/ongoing education

2. Did this session enhance your knowledge of the LGBTQ (Q-stands for questioning) identities and community?"

3. Did this session increase your overall awareness of disparate health problems within this community? 
4. Did this session enhance your knowledge of barriers to healthcare specific to this population?

5. Did this session increase your sensitivity and compassion for LGBTQ patients?

6. Overall, what do you rate this session?

7. After this presentation, do you feel more confident working with this population?

8. Did you self-select or were you placed in this session?

9. Rank your overall engagement in the presentation

10. What was the most valuable information you learned in this session?

11. Any additional questions/comments? 


\section{DATA ANALYSIS}

Quantitative data analysis was obtained using SPSS (SPSS, 2012) version 21.0. The frequencies and percentages, or means, along with the standard deviations were reported for all analysis. Using the Mann Whitney U test, the Second Life ${ }^{\mathrm{TM}}$ and Traditional instructional group were compared. All $\mathrm{p}$ values were two-tailed. The significant difference was set by convention at $p<0.05$. 


\section{RESULTS}

\section{PRE-SESSION SURVEY RESULTS}

The pre-session survey served to give an idea of the students' attitudes toward the LGBT community before attending the session. There were six questions on the pre-session survey that assessed students' feelings of the LGBT community before attending any of the Cultural Competency sessions.

In question 5 of the pre-session survey, students were asked to assess if they agreed or disagreed with the following statement, "access to health care is the same for LGBT persons as for other members of the population". Results of this question showed $54.1 \%$ of the student population either agreed or strongly agreed with this statement and $19.4 \%$ disagreed or strongly disagreed with that statement and $26.5 \%$ were uncertain (FIGURE 7).

Questions 8 and 11 sought to assess students' perceived knowledge of practices within the LGBT community. Question 8 on the pre-session survey asked students to evaluate whether they agreed or disagreed with the following assertion, "Most LGBT young people do their first "coming out" to a nonparent adult (e.g. a teacher or doctor)." The results showed that $55.7 \%$ of students 
either agreed or strongly agreed with this statement, $8.5 \%$ disagreed or strongly disagreed, and $35.8 \%$ of students were unsure (FIGURE 8 ).

Question 11 of the pre-session survey asked the students to access the longevity and monogamy of LGBT persons by having them rate if they agreed or disagreed with the following opinion, "LGBT people are less likely than heterosexual people to be in long-term monogamous relationships." Most students disagreed with this statement with $54.9 \%$ either disagreeing or strongly disagreeing. Only $14.1 \%$ of the student population agreed or strongly agreed with that statement and $31.0 \%$ were uncertain (FIGURE 9).

Questions 16, 21, and 26 dealt with the students addressing the questions as future physicians. To evaluate the students understanding of the healthcare needs of the LGBT community, the students were asked in Question 16 to agree or disagree with the statement that "LGBT patients have a unique set of health risks and health needs." The data collected found $45.9 \%$ of students felt that the LGBT community had a unique set of health risks and needs, while, $22.8 \%$ were uncertain. Overall, the majority of the students felt access to healthcare is the same for LGBT patients and members of other communities with over $30 \%$ of students strongly agreeing and $25.4 \%$ agreeing with that statement (FIGURE 10).

Many students showed that they understood the importance of knowing the sexual orientation of their patients, as evidenced in the results of Question 21. Results showed $37.9 \%$ agreeing and $17.5 \%$ strongly agreeing to the following declaration "As a healthcare provider I feel it is important for me to know about 
my patient's sexual orientation, sexual practices, and gender identity". Surprisingly, there were $5.6 \%$ who strongly disagreed and $18.3 \%$ who disagreed with that statement (FIGURE 11).

Question 26 assessed if students were comfortable treating members of the LGBT community by asking the students to determine if they agree or disagree with the statement "I would prefer not to treat patients with gender issues." The results of the pre-session survey showed that $14.7 \%$ of students were uncertain if they would prefer not to treat patients with gender identity issues. A great majority of the students, $80 \%$, stated that they disagreed or strongly disagreed with that statement (FIGURE 12). 


\section{POST-SESSION SURVEY RESULTS}

The hypothesis of this research project was that Second Life ${ }^{\mathrm{TM}}$ will enhance student interest in and mastery of the sensitive nature of LGBT disparities in the healthcare industry compared to the traditional session setting. The results of the data taken from the post-session interviews of both groups support this hypothesis.

There were eleven questions asked on the post-session survey administered to both the Traditional and Second Life ${ }^{\mathrm{TM}}$ LGBT group. Of the eleven questions, only two of the questions indicated a statistical difference, but the analysis of the other questions is pertinent as well. When averaging the overall score of four of the questions ( increase in sensitivity, increase in awareness of disparate health problems, enhanced knowledge of healthcare barriers, and enhanced knowledge of identities and communities), a significant difference was found between the Second Life ${ }^{\mathrm{TM}}$ group and the Traditional group (FIGURE 13).

The first question on the survey asked students to rank in order the four components of the session (blanket exercise, scenarios, reflection, and discussion of where to find resources/on-going education) from (4) most important to (1) least important. Interestingly, the results show that the Second Life $^{\mathrm{TM}}$ group had an average ranking of 3.13 for the scenarios and the Traditional 
group ranked the scenarios with an average score of 2.51. Also, the Second Life $^{\mathrm{TM}}$ group gave an average ranking of 3.13 for the blanket exercise, while the Traditional group gave an average ranking of 2.62 (TABLE 1).

Questions 2-6 and 9 asked the students to rank on a scale of 1 (being poor) to 5 (being excellent). Out of the six questions efficacy of the Second Life ${ }^{\mathrm{TM}}$ enhanced session compared to the Traditional workshop setting, only questions 3 and 5 showed significant differences (TABLE 2).

Question 2 asked "Did this session enhance your knowledge of the LGBTQ identities and community?" The results indicated that there was a slightly higher percent of students from the Second Life ${ }^{\mathrm{TM}}$ group, $88 \%$, that ranked this question a 4 or 5 compared to the Traditional group that only had $75 \%$ of students rank the session as increasing their knowledge of the LGBT community with a 4 or 5 .

In question 3 of the survey students were asked to evaluate how they felt about the question "Did this session increase your overall awareness of disparate health problems within this community?". All of the students in the Second Life ${ }^{\mathrm{TM}}$ group recorded a response of 4 or 5 . The Traditional group had $73 \%$ of students rank the question with a 4 or 5 . This question was found to have significant results (TABLE 2).

Question 4 of the survey asked "Did this session enhance your knowledge of barriers to healthcare specific to this population?" The results of the statistical analysis showed that the Second Life ${ }^{\mathrm{TM}}$ group had $100 \%$ of students give a 
response of 4 or 5 , whereas the Traditional group had $82 \%$ of students rank their response as 4 or 5 .

Question 5 of the survey asked students "Did this session increase your sensitivity and compassion for LGBTQ patients?" Results showed $100 \%$ of the Second Life ${ }^{\mathrm{TM}}$ group ranked the session with a 4 or 5 . While only $81 \%$ of the Traditional group indicated a response of 4 or 5 . This question showed a significant difference. Question 6 asked the students the overall rank of the session and again the Second Life ${ }^{\mathrm{TM}}$ group had $100 \%$ of students rank the session with a 4 or 5 . The Traditional group had $93 \%$ of students rank the session with a four or five (TABLE 2).

In question 7 the students were asked to give a response of yes, no, or uncertain to the question "After this presentation, do you feel more confident working with this population?". 100\% of the Second Life ${ }^{\mathrm{TM}}$ group indicated yes, compared to only $85 \%$ of the Traditional group (TABLE 2).

Question 9 asked students to rank their overall engagement in the presentation. Student responses showed that in the Second Life ${ }^{\mathrm{TM}}$ group $100 \%$ of respondents gave a 4 or 5 ranking and in the Traditional group $93 \%$ of respondents gave a 4 or 5 ranking (FIGURE 14). 


\section{FINDINGS FROM THE POST-SESSION SURVEY OPEN-ENDED QUESTIONS}

Two open-ended questions were asked on the post-session survey after both the Traditional and Second Life ${ }^{\mathrm{TM}}$ sessions of the LGBT workshops.

Question 10 of the post-session survey asked "What was the most valuable information you learned in this session?". Several themes were identified from the open-ended responses. There were 28 responses that indicated welcoming LGBT patients (presence of stickers or flags), providing a safe environment, and addressing barriers with patients as the most valuable information the students learned. There were twelve students that indicated that the information most valuable to them was learning how to make the medical intake forms unbiased and to not make assumptions during the patient history taking. Seven responses indicated that they felt learning about the increased health issues of the LGBT community was the most valuable information they learned from Second Life ${ }^{\mathrm{TM}}$

Question 11 of the post-session survey asked the students for any additional questions or comments they had about the LGBT community. Only six responses total were given from the attendees of both the traditional and Second Life $^{\mathrm{TM}}$ groups. One or more respondents were interested in knowing more about the following: perception the faith-based community has on the LGBT community, what the University of Louisville does, and Healthy People 2020. Three respondents offered kind comments toward the LGBT community stating, 
"The LGBT community is nice," "I'm an ally", and "looking to do outreach with the LGBT community".

When comparing the overall responses of the Second Life ${ }^{\mathrm{TM}}$ group to the Traditional group, the Second Life ${ }^{\mathrm{TM}}$ group significantly ranked the session with a higher score. 


\section{DISCUSSION}

\section{STUDENTS' PRE-EXISTING ATTITUDES AND FEELINGS TOWARD THE LGBT COMMUNITY AND KNOWLEDGE OF THEIR SPECIFIC HEALTHCARE NEEDS}

The results of our pre-session survey showed that while students possessed positive thoughts about the LGBT community, they lacked adequate knowledge about the LGBT community as well as an understanding of their specific healthcare needs. From the pre-session survey, we concluded that many students were still unsure and had a false sense of knowledge about the LGBT population, as well as a lack of sufficient knowledge to make definitive conclusions about the health care needs of members of the LGBT population. Students falsely strongly agreed with statements that they felt were true but, in fact research shows them to be untrue about the LGBT population. This was further observed in the post-survey open-ended questions. Students raised various questions about the LGBT population, such as, the relationship between the LGBT population regarding the faith-based community, how the University of Louisville is involved in promoting the well-being of the LGBT community, and 
what the Center of Disease Control (Healthy People 2020) is doing to change the health disparities that disproportionately affect the LGBT community.

The post-survey questions also showed uncertainty amongst the students. Each question had a high percentage of students that indicated a response of "uncertain or unsure" about the facts of the LGBT community. This shows that there is a greater need for the education of future healthcare professionals on the unique heath needs and lifestyle differences of the LGBT community. Some studies show that the reason students lack an understanding of the LGBT culture is that they also lack an understanding of what exactly encompasses one's culture (Dogra and Karnik, 2003).

In a questionnaire given to first-year medical students at the University of Illinois Colleges of Medicine at Chicago and Urbana-Champaign, it was found that students had an overall misunderstanding of culture as a whole (Dogra and Karnik, 2003). The majority of the students in this study, 87 out of 95, indicated that they thought of culture as a one dimensional concept (Dogra and Karnik, 2003). Students failed to see the complexity of the components that make up one's culture and how that would affect healthcare risks and concerns. One of the most important concepts of LGBT healthcare is recognizing that LGBT is a culture within itself. Not only is LGBT a culture, but its members have the additional challenge of dealing with stigmatism from their sexual orientation and for some from their gender and ethnicity as well. Therefore, the LGBT community has the added burden of placing an additional minority label on its members in addition to the minority labels that are within the normal parameters of society. 
Despite the lack of knowledge of the LGBT community and culture as a whole, our pre-session survey also identified that students had low levels of phobia and that the majority of students were not hesitant to work with the LGBT community. There are several previous studies that mirror these results for not only the LGBT population, but also various minority cultures as a whole (Dinkel et al., 2007; Dogra and Karnik, 2003). In 2002, a study involving nursing students showed similar results. Seventy-eight percent of the nursing students that participated in a survey that accessed attitudes on the LGBT community reported that they felt comfortable dealing with bisexuals or homosexuals (Peate et al., 2002). Other similar studies with both nursing students and social work students found that homophobia was low amongst students and that students possessed positive attitudes toward the LGBT community (Dinkel et al., 2007; Logie, 2008). This is vastly different from results seen in studies done with medical school students in prior decades. In a 1987 study with second and third-year medical students, students were randomly given clinical vignettes. After working through the symptoms, it was revealed to the students whether the patient had leukemia or AIDS (Kelly, 1987). The study found that both the AIDS patients and the homosexual patients were evaluated much more harshly and negatively. Some students even went so far as to say the homosexual patients deserved what that got (Kelly, 1987). Speculation as to why the students in 1987 viewed homosexuals so harshly compared to the students of the 2000 s would point to the lack of social acceptance of homosexuals of the time period. Now with an estimation minimum 10\% of Americans being homosexuals (Lim, 2013), one can 
hypothesize that acceptance from society has increased and changed societal attitudes thus having a positive influence on the students of this millennium.

In a previous study done by Rondahl in 2009 at a Swedish university, students showed inadequate knowledge about LGBT persons. Using a modified version of the survey, Knowledge about Homosexuality Questionnaire (Harris et al., 1995), medical and nursing students were asked to assess their knowledge of LGBT persons. It was found that $82 \%$ of the nursing and medical students at the university lacked knowledge essential for providing comprehensive care to LGBT patients (Rondahl, 2009). Similarly, a study done previously with Austrian medical students in 2004 showed that they also had a lack of knowledge of the LGBT population (Arnold et al., 2004). Medical students in this survey had uncertainties about the origin of homosexuality as well as the misbelief that homosexuals actually desire to be heterosexual, but simply need additional guidance. Such blatant misunderstanding of the LGBT issues leads to discrimination and poor healthcare practices. However, this trend of students with scarce knowledge about the unique disparities facing the LGBT community is not limited to individuals pursuing careers in medical and nursing fields. Other studies done in varying healthcare fields, both medical and social health, have shown similar results. In 2004, psychologists who were members of the National Association of School Psychologists were surveyed and evaluated on their attitudes, beliefs and knowledge of the LGBT population (Savage et al., 2004). Results of this study showed that the psychologists had low to moderate levels of 
knowledge about this population while still endorsing a positive attitude about the LGBT community as a whole (Savage et al,. 2004).

The field of social work has shown similar results in evaluating students' readiness to serve the LGBT community. A study in 2008 set out to evaluate the phobias, attitudes, and overall cultural competence of social work students demonstrated that $15.6 \%$ of students were not ready or were not sure if they were ready to advocate on behalf of the LGBT community (Logie, 2008). Even more shocking was the fact that $42.4 \%$ of the students who took part of the selfassessment survey said they were not sure of the challenges facing the LGBT population (Logie, 2008). Based on these various studies it is not surprising that the government has made LGBT healthcare a priority in its Healthy People 2020 initiative (Healthy People 2020).

The exact reason why specific knowledge of the LGBT community is lacking in students is unknown. Research points to the low number of curriculum hours dedicated to training students on the plight of the LGBT community (Butler, 2010; Sanchez et al. 2006; Sequeira et al., 2012; Tamas et al., 2010). A study of preclinical medical students at Tulane University indicated that a key concern of students was a lack of exposure to LGBT content (Sequeira et al., 2012). Students were asked to self-report what they felt was lacking from the normal medical school curriculum related to the LGBT population. The responses from the students indicated a desire to know more about the LGBT community and a dislike of the lack of time and dedication to the LGBT community in their medical school studies (Sequeira et al., 2012). 
Each year students graduating from medical school are asked to complete a questionnaire from the AAMC. In 2010 , results of that survey showed that $29 \%$ of the students at the University of Louisville felt that the amount of time dedicated to human sexuality was inadequate. A follow-up study was done to assess the amount of time within the curriculum actually dedicated to human sexuality (Tamas et al., 2010). At the University of Louisville approximately 6 hours of instructional time was dedicated for human sexuality, which is twice the average amount recommended by the American Medical Student Association Plus One Initiative Report. (Tamas et al., 2010). Course directors were reported to feel that eleven hours of instructional time was more than ideal, but many course instructors found human sexuality irrelevant to the topics they were teaching (Tamas et al., 2010). In the same study, it was found that instructors who identified the most barriers to teaching LGBT issues had the lowest amount of instructional time dedicated to the instruction of those issues (Tamas et al., 2010). These studies, as well as the results of our study, indicate that there is a need for training of instructors in the incorporation of LGBT issues within their current curriculum. Training instructors on how to teach LGBT disparities will provide students with a solid foundation of LGBT disparities and their specific healthcare needs which are essential in developing quality healthcare providers. We also see from both our study and the study done by Tamas et al. at the University of Louisville that there is a desire from both the students and the faculty to be better trained in healthcare concerns as it relates to human sexuality. However, an effective teaching tool is needed to aid students and 
potentially instructors as well. It is our belief that Second Life ${ }^{\mathrm{TM}}$ can serve as that teaching tool. 


\section{SECOND LIFE ${ }^{T M}$ AS A TOOL TO INCREASE STUDENT ENGAGEMENT, SENSITIVITY, AND AWARENESS}

Questions 3 and 5 of the post-survey showed significant differences. The two questions were:

Did this session increase your sensitivity and compassion for LGBT patients?

Did this session increase your awareness of disparate health problems within this population?

Upon initial analysis of the two questions, one can identify that both questions pertain to with feelings/empathy toward the LGBT community. It is also noteworthy that in the two questions that demonstrated statistical significance that there were students from the Traditional group who ranked their overall sensitivity and awareness after being in the session as poor. There were no students from the Second Life ${ }^{\mathrm{TM}}$ group that ranked those questions with a poor review in the post-session survey.

Our study utilized a validated tool in teaching cultural competence and communication skills for healthcare professionals by using the scenario vignettes. Not only had the scenario vignettes been used in previous year workshops, but in addition it had been used for many years throughout the 
medical training of students (Kelly, 1987). We took an already proven method and set out to improve it by incorporating the vignettes into Second Life ${ }^{\mathrm{TM}}$. From our results, Second Life ${ }^{\mathrm{TM}}$ is an effective tool to increase student mastery of culturally sensitive topics to increase student awareness, and to increase student confidence in those sensitive subject matters. Our study shows that students responded more positively to the Second Life ${ }^{\mathrm{TM}}$ enhanced LGBT session than the traditional lecture session. From the results, we observed that the students that attended the Second Life ${ }^{\mathrm{TM}}$ session had an increase in sensitivity and compassion as well as, an increase in the awareness of the health disparities of the LGBT population.

One could speculate that the Second Life ${ }^{\mathrm{TM}}$ format allowed the students to a put a face and a voice to the patient compared to the Traditional program. In addition Second Life ${ }^{\mathrm{TM}}$ has been found to be aesthetically pleasing and could potentially increase student engagement (Hussainy et al., 2012). This is not the first time that Second Life ${ }^{\mathrm{TM}}$ has been proven to improve student interest in and mastery of a specific study topic (Richardson, 2011; Yellowlees and Cook, 2006). The interaction in our Second Life ${ }^{\mathrm{TM}}$ scenarios sparked a real-life effect amongst the students and likely their level of empathy was increased. Perhaps putting a face to the discriminatory action, even if only a virtual one, appealed to the humanity of the students and made it more realistic. On the other hand, in the Traditional group, students only had scenarios written on a piece of paper and had no true connection with the characters from the scenario. One can hypothesize that since the Second Life ${ }^{\mathrm{TM}}$ group was able to observe the scenario 
in front of them they were more actively engaged in the session. Second Life ${ }^{\mathrm{TM}}$ has the benefit of providing students with visualization, contextulazation, and identification of various content (Warburton, 2009). The freedom of Second Life $^{T M}$ is the ability to create whatever environment you see fit and by doing so, provide students with a more authentic experience. Second Life ${ }^{T M}$ has also been shown in other studies to invoke empathy toward a certain population of society (Boellstorff, 2008). In addition, the scenarios in Second Life ${ }^{\mathrm{TM}}$ provided a voice to the victim. Hearing the interactive account of discrimination likely had a more profound effect toward empathy amongst students than to have a discriminatory issue read to them.

In 2010, John Wiecha, reported that Second Life ${ }^{\mathrm{TM}}$ was found to have a positive influence on engagement and attention (Weicha, 2010). In his study, Weicha set out to evaluate the use of Second Life ${ }^{\mathrm{TM}}$ in postgraduate medical education for primary care physicians working with insulin dependent patients. Overall, the participants in the study found Second Life ${ }^{\mathrm{TM}}$ to be more engaging than a traditional lecture setting and found it to be a superior learning tool compared to other online trainings (Weicha, 2010). An increase in confidence regarding insulin therapy was also indicated from the results (Weicha, 2010). This correlates with our results with $100 \%$ of the Second Life ${ }^{\mathrm{TM}}$ group reporting to be more confident in working with the LGBT population. In addition, Weicha's study showed that Second Life ${ }^{\mathrm{TM}}$ could be used to impact clinical competence positively. There were two important advantages of Second Life ${ }^{\mathrm{TM}}$ that were indicated by the participants and that was the sense of presence afforded to 
them by the avatars and the real-life application provided by the mock patients (Weicha, 2010).

In another study involving paramedic workers, Second Life ${ }^{\mathrm{TM}}$ was found to be a useful method in teaching complex scenarios. Ninety-six percent of the paramedic workers that took place in the emergency preparedness seminar within Second Life ${ }^{\mathrm{TM}}$ found it to be a useful teaching method and $91 \%$ found it enjoyable (Cohen et al., 2013). Moreover, 95\% of the participants would use a similar simulation again for additional training (Cohen et al., 2013). It was also noteworthy that the participants felt that the use of Second Life ${ }^{\mathrm{TM}}$ would allow for training in difficult or impossible in real life environments (Cohen et al., 2013), similar to our emergency room scenario.

While Second Life ${ }^{\mathrm{TM}}$ is an effective tool to increase student awareness some argue that Second Life ${ }^{\mathrm{TM}}$ could also be a distraction. In fact some students have indicated in certain studies that some of the uncontrollable environmental aspects inside of Second Life ${ }^{\mathrm{TM}}$ can be distracting, such as, the ability to fly around, distractive costumes, and local chat (Weicha et al. 2010; Petrakou, 2010). However, this distraction is no more than what could be experienced in a normal classroom setting (Petrakou, 2010). It is evident that Second Life ${ }^{\mathrm{TM}}$ should be considered a tool for medical education, especially with cultural sensitive information. 


\section{SECOND LIFE ${ }^{\mathrm{TM}}$ AS A TOOL TO INCREASE STUDENT CONFIDENCE AND IMPROVE COMMUNICATION SKILLS}

Second Life ${ }^{\mathrm{TM}}$ gives the distinct advantage of anonymity (Annang et al., Baker et al., 2009; Richardson et al., 2011). Within Second Life ${ }^{\mathrm{TM}}$, users have a blanket of concealment which promotes self-confidence, openness, and engagement. There is a sense of protection that comes from the use of avatars (Weicha, 2010). This is important within the context of studying of culturally sensitive material such as LGBT disparities.

Second Life ${ }^{\mathrm{TM}}$ has been found to be an effective education tool in improving dialogue between doctors and mock patients. In 2011, Mitchell et al. developed a pilot study to evaluate Second Life ${ }^{\mathrm{TM}}$ as a tool to improve patientcentered communication (Mitchell et al., 2011). Seventy-seven percent of the participants found that Second Life ${ }^{\text {TM }}$ was an effective educational medium for helping to improve dialogue between provider and patient (Mitchell et al., 2011). Other studies have also indicated that Second Life ${ }^{\mathrm{TM}}$ is an effective tool to improve communication skills, and allow for openness (Conradi, 2009; Kidd et al., 2012; Hussainy et al., 2012; Mitchell et al., 2011; Twining, 2009). The pseudoanonymity afforded by the avatars in Second Life ${ }^{\mathrm{TM}}$ makes for a comfortable learning environment, as reported by students in a pilot study at the University of Kentucky (Richardson et al., 2011). Student participation was found 
to have increased when compared to a traditional lecture environment (Richardson et al., 2011). Within Second Life ${ }^{\mathrm{TM}}$, students have the luxury of trial and error without the fear of judgment. Second Life ${ }^{\mathrm{TM}}$ has been found to build confidence with the use of mock patients before actually having to put those practices to work in real life (Sweigart and Hodson-Carlton, 2013). 


\section{THE USE OF VIRTUAL SIMULATION AS TEACHING METHOD FOR LGBT HEALTHCARE}

While homophobia has dwindled in recent years, it still exists. To overcome those barriers, clear, non-biased teaching strategies must be incorporated to produce well-rounded competent physicians. Research has found that simulation is one solution to overcome the limitations of traditional clinical education (Lim, 2013.) Lim suggests training specific standardized patients to have LGBT identities (Lim, 2013).Lim argues that diverse clinical education provides a well-rounded clinical education (Lim, 2013). From our study, we believe that Second Life ${ }^{\mathrm{TM}}$ can provide a venue for standardized patients in the form of avatars. As suggested by Lim, the avatars can be programmed to have a specific LGBT identity and be used as a tool to aid future healthcare workers; as well as current healthcare workers, to develop proper communication skills to deal with the LGBT community, such as not assuming heterogeneity and appropriate use of the words "gender' and 'sex" (Coren, 2011).

Virtual worlds allow for cultural sensitive education (Games and Bauman, 2011). One of the aims of this study was to increase student consciousness of avoiding the use of heterosexual bias in language while taking the medical history reports from a patient. In our study, Second Life ${ }^{\mathrm{TM}}$ was used as a tool to highlight the bias that many patients encounter while giving their medical history 
to healthcare providers. In one study done by Hussainy et al., involving pharmacy students, a virtual practice environment was used an educational tool to assess the dialogue between pharmacists and patients (Hussainy et al., 2012). The virtual practice environment allowed for a safe, risk-free environment that allowed students to develop their communication skills and professional acculturation (Hussainy et al., 2012; Kidd et al., 2012). In that study, 90\% of participants indicated that they had an improvement in communication skills after attending the training (Hussainy et al., 2012). In another study involving nursing students, researchers used virtual environments to enhance interview skills between nurses and patients for urological, genital, and sexual subjective assessments (Sweigart and Hodson-Carlton, 2013). Students that participated in the virtual environment training were found to give more thorough subsequent health interviews following the training (Sweigart and Hodson-Carlton, 2013). In addition, the number of questions asked by the nurse to the mock patients increased with those students that attended the training (Sweigart and HodsonCarlton, 2013).

It has been suggested by the AAMC to use e-learning as a tool to teach physicians about LGBT issues (AAMC, 2005). One subclass of e-learning is the use of virtual worlds. It has been found that the use of virtual worlds allows for cultural-sensitive education (Games and Bauman, 2011). One specific subgenre of LGBT is transgendered patients. Studies have shown that training specifically for transgendered patients is needed (Lurie, 2005). Transgendered patients are 
one of the most poorly understood subgenres of LGBT health and new innovative ways to teach healthcare risks for this community is needed (Lurie, 2005).

Another benefit of virtual world is confidentiality. The computer screen acts as protector from the outside world. It is important when dealing with LGBT health issues that students have a venue where they can speak openly without risks of scrutiny or judgment. Ensuring confidentiality is important for the safety and privacy of those participating in studies or seminars on LGBT health (Mayer et al., 2008). E-learning, such as in Second Life ${ }^{\mathrm{TM}}$, allows for a student to build on their confidence before actually having to put their knowledge into practical application (Sweigart and Hodson-Carlton, 2013).

Another benefit to Second Life ${ }^{\mathrm{TM}}$ is the ability to construct scenarios that are difficult or impossible to do in real life (Boulos, 2007; Cohen, 2013). In Second Life ${ }^{\mathrm{TM}}$ users have the ability to navigate through the virtual world in various ways such as, flying, teleporting, riding in various vehicles (Boulos, 2007). Transportation in Second Life ${ }^{\mathrm{TM}}$ is quicker than in real life and provides for a wider range of classroom space for use. In addition, impractical or hard to reproduce scenarios can be made possible in Second Life ${ }^{\mathrm{TM}}$, like the emergency room scenario that was presented in this study (Coren, 2011). Second Life ${ }^{\mathrm{TM}}$ offers the ability of customization. Scenarios can be tailored to meet a classroom or educational discipline's specific need. The possibilities in Second Life ${ }^{\mathrm{TM}}$ are endless.

Finally, Second Life ${ }^{\mathrm{TM}}$ offers the benefit of anytime learning. This allows for distance learning, across college campuses, and even potentially across the 
world (Boulos, 2007; Richardson, 2011). Due to virtual users being able to log on in Second Life ${ }^{\mathrm{TM}}$ from any compatible computer, it allows for a diverse set of students and a diverse set of instructors. In relation to this study, members of the LGBT population can be virtual standardized patients allowing for a more authentic patient-physician interaction. 


\section{LIMITATIONS OF OUR STUDY}

The limitations of this study are similar to those seen in other case studies involving Second Life ${ }^{\mathrm{TM}}$. One of the limitation was the small study size due restricted admission due to this being a pilot study. The small size could have influenced the results because the students who attended both sessions were already interested in learning about the LGBT community. Also, the small size of students could have shown bias in the open-ended responses that were given.

In addition, the need for technical support staff to troubleshoot technical issues in a timely fashion without affecting the realism of the project was also a concern and hindered the use of Second Life ${ }^{\mathrm{TM}}$ by the attendees of our Second Life $^{\mathrm{TM}}$ session. Lastly, the learning curve and time allotment to teach volunteer, as well as students, the Second Life ${ }^{\mathrm{TM}}$ navigation system was a limitation in this study. 


\section{FUTURE WORK}

In the future, it is our hope that attendees of the Second Life ${ }^{\mathrm{TM}}$ workshop will be able to log into Second Life ${ }^{\mathrm{TM}}$ with their individual avatars. By logging in with their own personalized avatars, the students will have the option to either participate as the doctor or the LGBT patient seeking treatment. The students will have prompts that will allow them to choose from a number of choices in how to proceed through each scenario. If a culturally insensitive choice is made, the students will be corrected and hopefully will learn better communication skills with the LGBT patients and not make the same mistake when they put their skills learned into real practice.

When looking at the current study and the study by Tamas et al, we see that there is a greater need for education of our medical students on the disparities facing the LGBT community. In the future, it would be interesting to take a look at the AAMC graduate questionnaire results of University of Louisville medical school graduates who participated in the Second Life ${ }^{\mathrm{TM}}$ enhanced LGBT session. A comparison of previous results will give an indication on whether the Second Life ${ }^{\mathrm{TM}}$ enhanced session increased students awareness of the LGBT population, as well as provided a greater sense of preparedness when working in the future with the LGBT community. 


\section{CONCLUSION}

Based on the results of our study, more instructional time is needed to increase student knowledge of the specific disparities that face the LGBT population. In addition, it was promising to see that overall the majority of students have a positive connotation about the LGBT community as a whole. More importantly, we showed that Second Life ${ }^{\mathrm{TM}}$ is an effective tool to increase student mastery of the sensitive nature of the health disparities facing the LGBT community as well as an effective tool to increase student engagement and interest in the LGBT population. 


\section{REFERENCES}

Association of American Medical Colleges. Cultural Competence Education for Medical Students. Washington: AAMC; (2005).

Annang, Lucy, Jessica L. Muilenburg, and Sheryl M. Strasser. "Virtual worlds:

Taking health promotion to new levels." American Journal of Health

Promotion 24.5 (2010): 344-346.

Baker, Suzanne C., Ryan K. Wentz, and Madison M. Woods. "Using virtual worlds in education: Second Life ${ }^{\circledR}$ as an educational tool." Teaching of Psychology 36.1 (2009): 59-64.

Bainbridge, William Sims. "The scientific research potential of virtual worlds." Science 317.5837 (2007): 472-476.

Balint, Enid. "The possibilities of patient-centered medicine." The Journal of the Royal College of General Practitioners 17.82 (1969): 269.

Barker, M. R. "Gay and lesbian health disparities: Evidence and recommendations for elimination." Journal of Health Disparities Research and Practice 2.2 (2012): 6.

Bauman, Eric B. Game-Based Teaching and Simulation in Nursing and Health Care. Springer Publishing Company, 2012.

Beach, Mary Catherine, Somnath Saha, and Lisa A. Cooper. The role and relationship of cultural competence and patient-centeredness in health care quality. Vol. 36. Commonwealth Fund, 2006.

Beagan, Brenda L. "Teaching Social and Cultural Awareness to Medical Students:" It's All Very Nice to Talk about It in Theory, But Ultimately It Makes No Difference"." Academic Medicine 78.6 (2003): 605-614.

Beamon, Carmen J. A guide to incorporating cultural competency into health professionals' education and training. Diss. School of Medicine, School of Public Health Janelle A. Shumate, University of North Carolina, 2006. 
Beard, Leslie, Kumanan Wilson, Dante Morra, and Jennifer Keelan. "A survey of health-related activities on second life." Journal of Medical Internet Research 11 , no. 2 (2009).

Berlin, Elois Ann, and William C. Fowkes Jr. "A teaching framework for crosscultural health care-application in family practice." Western Journal of Medicine 139.6 (1983): 934.

Betancourt, Joseph R., Alexander R. Green, and J. Emilio Carrillo. Cultural competence in health care: emerging frameworks and practical approaches. Vol. 576. Commonwealth Fund, Quality of Care for Underserved Populations, 2002.

Boellstorff, Tom. Coming of age in Second Life [electronic resource]: an anthropologist explores the virtually human. Princeton University Press, 2008.

Boulos MN, Hetherington L, Wheeler S. Second Life: an overview of the potential of 3-D virtual worlds in medical and health education. Health Info Library Journal. 2007 Dec;24(4):233-45.

Boutin-Foster, Carla, Jordan C. Foster, and Lyuba Konopasek. "Viewpoint: physician, know thyself: the professional culture of medicine as a framework for teaching cultural competence." Academic Medicine 83.1 (2008): 106-111.

Brach, Cindy, and Irene Fraserirector. "Can cultural competency reduce racial and ethnic health disparities? A review and conceptual model." Medical Care Research and Review 57.4 suppl (2000): 181-217.

Bruessow, Diane, and D. F. A. A. P. A. rPA-c. "Keeping up with LGBT health: why it matters to your patients." JAAPA: Official journal of the American Academy of Physician Assistants 24.3 (2011): 14.

Butler, Rachel A. "An Assessment of Lesbian, Gay, Bisexual, and Transgender Curriculum Infusion in US Medical Schools." (2010).

Campinha-Bacote, Josepha. The process of cultural competence in the delivery of healthcare services: A culturally competent model of care. Transcultural CARE Associates, 2003.

Chapman, Suzzane, Bates, T., O'Neil, E. and, Chan, M. 2008. Teaching Cultural Competence in allied health professions in California. Centre for the Health Professions. University of California (SF).

Clark, Lauren, Jeannie Zuk, and Julaluk Baramee. "A literary approach to teaching cultural competence." Journal of Transcultural Nursing 11.3 (2000): 199-203. 
Cohen, Daniel, Nick Sevdalis, David Taylor, Karen Kerr, Mick Heys, Keith Willett, Nicola Batrick, and Ara Darzi. "Emergency preparedness in the 21st century: Training and preparation modules in virtual environments." Resuscitation 84, no. 1 (2013): 78-84.

Coren JS, Coren CM, Pagliaro SN, Weiss LB. Assessing your office for care of lesbian, gay, bisexual, and transgender patients. The Healthcare Manager. 2011 Jan-Mar;30(1):66-70.

Cross TL, Bazron BJ, Dennis KW, Isaacs MR. Towards a Culturally Competent System of Care: A Monograph on Effective Services for Minority Children Who Are Severely Emotionally Disturbed. Vol 1. Washington, DC:Georgetown University, National Technical Assistance Center for Children's Mental Health; 1989.

Crandall, Sonia J., Geeta George, Gail S. Marion, and Steve Davis. "Applying theory to the design of cultural competency training for medical students: a case study." Academic Medicine 78, no. 6 (2003): 588-594.

Dinkel, Shirley, Brenda Patzel, Michael J. McGuire, Elaine Rolfs, and Kelly Purcell. "Measures of homophobia among nursing students and faculty: a Midwestern perspective." International Journal of Nursing Education Scholarship 4, no. 1 (2007).

Dogra, Nisha, and Niranjan Karnik. "First-year medical students' attitudes toward diversity and its teaching: an investigation at one US medical school." Academic Medicine 78.11 (2003): 1191-1200.

Durand, Cheryl, Cheryl Abel, Matthew Silva, and Alicia Desilets. "An elective course in cultural competence." Currents in Pharmacy Teaching and Learning 4, no. 2 (2012): 102-108.

Flores, Glenn, Denise Gee, and Beth Kastner. "The teaching of cultural issues in US and Canadian medical schools." Academic Medicine 75.5 (2000): 451455.

Foss, Jeremy. "Lessons from learning in virtual environments." British Journal of Educational Technology 40.3 (2009): 556-560.

Games, Alex Ivan, and Eric B. Bauman. "Virtual worlds: an environment for cultural sensitivity education in the health sciences." International Journal of Web Based Communities 7.2 (2011): 189-205.

Giddens, Jean Foret, Sarah North, Linnea Carlson-Sabelli, Erin Rogers, and Louis Fogg. "Using a Virtual Community to Enhance Cultural Awareness." Journal of Transcultural Nursing 23, no. 2 (2012): 198-204. 
Gorini, Alessandra, Andrea Gaggioli, Cinzia Vigna, and Giuseppe Riva. "A second life for eHealth: prospects for the use of 3-D virtual worlds in clinical psychology." Journal of medical Internet research 10, no. 3 (2008).

Hansen, Margaret M. "Versatile, immersive, creative and dynamic virtual 3-D healthcare learning environments: a review of the literature." Journal of Medical Internet Research 10.3 (2008).

Harris, Mary B., Jane Nightengale, and Nancy Owen. "Health care professionals' experience, knowledge, and attitudes concerning homosexuality." Journal of gay \& lesbian social services 2.2 (1995): 91-108.

Hawala-Druy, Hawala-Druy and Hill, and Mary H. Hill. "Interdisciplinary: Cultural competency and culturally congruent education for millennials in health professions." Nurse Education Today 32, no. 7 (2012): 772-778.

Hewitt, Anne M., Susan S. Spencer, Rameshsharma Ramloll, and Heidi Trotta. "Expanding CERC beyond public health: Sharing best practices with healthcare managers via virtual learning." Health Promotion Practice 9, no. 4 suppl (2008): 83S-87S.

Hoban, Mary T., and Robert L. Ward. "Building culturally competent college health programs." Journal of American college health 52.3 (2003): 137-141.

Huckabee, Michael J., and Gina S. Matkin. "Examining Intercultural Sensitivity and Competency of Physician Assistant Students." Journal of Allied Health 41.3 (2012): 55E-61E.

Hussainy, Safeera Yasmeen, Kim Styles, and Greg Duncan. "A Virtual Practice Environment to Develop Communication Skills in Pharmacy Students." American journal of pharmaceutical education 76.10 (2012).

Jarmon, Leslie. "Pedagogy and learning in the virtual world of Second Life." Encyclopedia of distance and online learning 3 (2008): 1610-1619.

Kaiser Family Found. 2001.Inside-Out: A Report on the Experiences of Lesbians, Gays and Bisexuals in America and the Public's View on Issues and Policies Related to Sexual Orientation, Menlo Park, CA.

Kelley, Leah, Calvin L. Chou, Suzanne L. Dibble, and Patricia A. Robertson. "A critical intervention in lesbian, gay, bisexual, and transgender health: Knowledge and attitude outcomes among second-year medical students." Teaching and Learning in Medicine 20, no. 3 (2008): 248-253. 
Kelly, Jeffrey A., Janet S. St Lawrence, Steve Smith Jr, Harold V. Hood, and Donna J. Cook. "Medical students' attitudes toward AIDS and homosexual patients." Academic Medicine 62, no. 7 (1987): 549-56.

Kelly, Patricia J. "Should we rethink how we teach cultural competency in physician assistant education?." The journal of physician assistant education: the official journal of the Physician Assistant Education Association 23.3 (2011): 42-45.

Kidd, Lori I., Samantha J. Knisley, and Karyn I. Morgan. "Effectiveness of a Second Life ${ }^{\circledR}$ Simulation as a Teaching Strategy for Undergraduate mental Health nursing Students." Journal of psychosocial nursing and mental health services 50.7 (2012): 28-37.

Kleinman A, Eisenberg L, Good B. Culture, illness, and care: clinical lessons from anthropologic and cross-cultural research. Ann Intern Med 1978; 88(2):251-258.

Kripalani, Sunil, Jada Bussey-Jones, Marra G. Katz, and Inginia Genao. "A prescription for cultural competence in medical education." Journal of General Internal Medicine 21, no. 10 (2006): 1116-1120.

Lamda Legal.When Health Care Isn't Caring: Lambda Legal's Survey on Discrimination Against LGBT People and People Living with HIV. 2009. Available: <http://data.lambdalegal.org/publications/downloads/whcicreport_when-health-care-isnt-caring.pdf>. Accessed: November 5, 2013.

Leavitt, R. "Developing cultural competence in a multicultural world: Part 1." Magazine of Physical Therapy 10.12 (2002).

Leininger M. Transcultural Nursing: Theories, Concepts and Practices. New York: Wiley; 1978.

Lim, Fidelindo, James C. Pace, B. S. N. Kimberly Bailey, and Henrietta Jones. "Caring for older lesbian, gay, bisexual, and transgender adults." Am Nurse Today 8, no. 1 (2013).

Long, Tracey B. "Overview of teaching strategies for cultural competence in nursing students." Journal of cultural diversity 19.3 (2011): 102-108.

Lurie, Samuel. "Identifying training needs of health-care providers related to treatment and care of transgendered patients: A qualitative needs assessment conducted in New England." International Journal of Transgenderism 8.2-3 (2005): 93-112. 
Lynch, Eleanor W., and Marci J. Hanson. Developing cross-cultural competence: A guide for working with young children and their families. Paul $\mathrm{H}$. Brookes Publishing, 1992.

Mayer, Kenneth H., Judith B. Bradford, Harvey J. Makadon, Ron Stall, Hilary Goldhammer, and Stewart Landers. "Sexual and gender minority health: what we know and what needs to be done." American Journal of Public Health 98, no. 6 (2008): 989-995.

McKay, Becky. "Lesbian, gay, bisexual, and transgender health issues, disparities, and information resources." Medical reference services quarterly 30.4 (2011): 393-401.

Mitchell, Suzanne, Robin Heyden, Neil Heyden, Paul Schroy, Stephen Andrew, Ekaterina Sadikova, and John Wiecha. "A pilot study of motivational interviewing training in a virtual world." Journal of medical Internet research 13, no. 3 (2011).

Mollon, L., Stahl, J. Providing the Best Care for LGBT Patients. 2010. http://studentdoctor.net/2010/07/providing-the-best-care-for-Igbt-patients/.

Kalbfleisch, Norm., and Schmidt, Terri. "Cultural Competency on Lesbian, Gay, Bisexual or Transgender (LGBT)."

Obedin-Maliver, J., Goldsmith, E.S., Stewart, L., White, W., Tran, E., Brenman, S, Wells, M., Fetterman, D.M., Garcia, G., Lunn, M.R. Lesbian, gay, bisexual, and transgender-related content in undergraduate medical education. JAMA 2011: 306(9):971-7.

$\mathrm{OMH}$, Office of Minority Health. National Standards on Culturally and Linguistically Appropriate Services (CLAS) in Health Care. Executive Summary. 2001. Washington, D.C., U.S. Department of Health and Human Services. http://minorityhealth.hhs.gov/templates/browse. aspx?|v|=2\&|v||ID=11

Parkhill, Amy L., Jeanne Gainsburg, Scott Fearing, and Jennifer L. Mathews. "The need for transgender health content in the pharmacy curriculum." Innovations in Pharmacy 2, no. 4 (2011): 58.

Peate, I., Suominen, T.,Valimaki, M., Lohrmann, C. \& Muinonen, U. (2002). HIV/AIDS and its impact on student nurses. Nurse Education Today, 22, 492501.

Petrakou, Alexandra. "Interacting through avatars: Virtual worlds as a context for online education." Computers \& Education 54.4 (2010): 1020-1027. 
Purnell, Larry. "The Purnell model for cultural competence." Journal of transcultural nursing 13.3 (2002): 193-196.

Richardson, April, Matthew Hazzard, Sandra D. Challman, Aaron M. Morgenstein, and Jennifer K. Brueckner. "A "Second Life" for gross anatomy: Applications for multiuser virtual environments in teaching the anatomical sciences." Anatomical sciences education 4, no. 1 (2011): 39-43.

Rondahl, Gerd. "Students' inadequate knowledge about lesbian, gay, bisexual and transgender persons." International journal of nursing education scholarship 6.1 (2009).

Ruiz, Jorge G., Michael J. Mintzer, and Rosanne M. Leipzig. "The impact of elearning in medical education." Academic medicine 81, no. 3 (2006): 207-212.

Rutledge, Carolyn M., Phyllis Barham, Lynn Wiles, Richardean S. Benjamin, Phyllis Eaton, and Kay Palmer. "Integrative simulation: A novel approach to educating culturally competent nurses." Contemporary Nurse 28, no. 1-2 (2008): 119-128.

Saha, Somnath, Beach, Mary Catherine and Cooper, Lisa A. Patient Centeredness, Cultural Competence and Healthcare Quality. 2008.

Sargent, Sara E., Carol A. Sedlak, and Donna S. Martsolf. "Cultural competence among nursing students and faculty." Nurse education today 25.3 (2005): 214-221.

Sequeira, Gina M., Chayan Chakraborti, and Brandy A. Panunti. "Integrating lesbian, gay, bisexual, and transgender (LGBT) content into undergraduate medical school curricula: a qualitative study." The Ochsner Journal 12.4 (2012): 379-382.

Simon, Melissa A., E. Chang, and XinQi Dong. "Partnership, reflection and patient focus: advancing cultural competency training relevance." Medical education 44.6 (2010): 540-542.

Singhal, Arvind, and Everett M. Rogers. Entertainment-education: A communication strategy for social change. Routledge, 1999.

Sweigart, Linda, and Kay Hodson-Carlton. "Improving student interview skills: the virtual avatar as client." Nurse educator 38.1 (2013): 11-15.

Tamas, Rebecca, Karen Hughes Miller, Leslee J. Martin, and Ruth B. Greenberg. "Addressing patient sexual orientation in the undergraduate medical education curriculum." Academic Psychiatry 34, no. 5 (2010): 342-345. 
Twining, Peter. "Exploring the educational potential of virtual worlds-Some reflections from the SPP." British Journal of Educational Technology 40.3 (2009): 496-514.

U.S. Department of Health and Human Services. Office of Disease Prevention and Health Promotion. Healthy People 2020. Washington, DC. Available at [http://www.healthypeople.gov/2020/topicsobjectives2020/overview.aspx?topi cid=25]. Accessed [June 30,2012].

Warburton, Steven. "Second Life in higher education: Assessing the potential for and the barriers to deploying virtual worlds in learning and teaching." British Journal of Educational Technology 40.3 (2009): 414-426.

Wiecha, John, Robin Heyden, Elliot Sternthal, and Mario Merialdi. "Learning in a virtual world: experience with using second life for medical education." Journal of medical Internet research 12, no. 1 (2010).

Yellowlees, Peter and James Cook. "Education about hallucinations using an internet virtual reality system: a qualitative survey." Academic Psychiatry 30.6 (2006): 534-539. 


\section{DMinsiti}

Making A World Of Difference In Healthcare

Learning Objectives:

- Understand the history of acupuncture and how it is used in western society

- Introduce students to cultural issues involved with the mennonite community

- How to work effectively with Lesbian, Gay, Bisexual or Transgendered (LGBT) patients whose beliefs systems may differ from your own

- Identify areas where Islamic principles could impact the practice of western medicine on musim patients. Develop/evaluate strategies for adjusting current medical protocols to respect islamic beliefs.

- Increase the knowledge of mental/ behavioral health issues and military culture of veterans/service members and their families

- Explore the intersection of legal child discipline with illegal child maltreatment along with the associated cultural implications

- Identify preconceived ideas that students bring to cultural competency and how they might be considered stereotypes
A special THANK YOU to our sponsors:

University of Louisville

School of Medicine, Interim Dean Toni Ganzel

School of Dentistry, Dean John Sauk

University of Louisville AHEC Central Office

University of Louisville School of Nursing

University of Louisville Cultural Center

University of Louisville- Student Government Association (SGA)

Uofl Commission on Diversity \& Racial Equality (CODRE)

Kentucky AHEC Regional Offices and Staff

Recognized Student Organization (RSO): HSC Cultural Competency Workshop Committee

Breakfast Provided by:

Lunch Provided by:

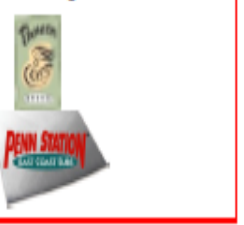

7 th Annual

University of Louisville Health Sciences center Cultural Competency Workshop

REGISTRATION INFORMATION:

The conference is opea at NO COST to U of L HSC students, haculty, and staff.

Students register through Blackboard- the site will appear on your menu if your class is schedulded to attend. If you have ques. tions contact Roa Welch at: aheconfonflocisville edu For aseessment \& evaluation contact Michael Rowland at: mlrowlo20. baisille.etu

Faculty and staff, register via emall to: Gloria Pressley-McGruder, EdD @ gjpres01@loulsville.edu

UNIVERSITY OF LOUISVILLE. SOMOOL OF MEACNI 7th Annual

University of Louisville

Health Sciences Center Cultural Competency Workshop

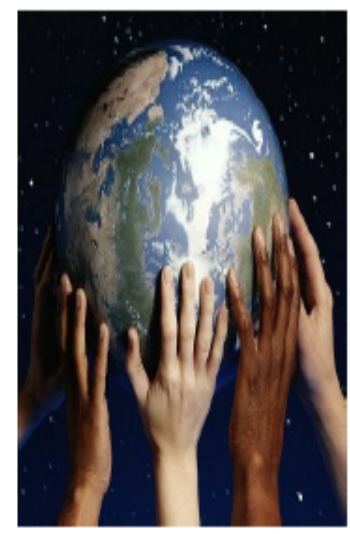

Increasing Cultural

Competency in

Our Changing World

University of Louisville Health Sciences Center

September 27, 2012

8:00 am - 2:30 pm

\section{FIGURE 1: 2012 Cultural Competency Workshop at the University of Louisville.}




\section{WORKSHOP AGENDA}

7:00 am-8:00 am- Registration/Brealefast

Kornhauser Library Lobby

8:00 am- 8:40 am- Welcome to Students

Dr. Mordean Taylor-Archer

Vice Provost for Diversity \& Intemational Affairs

University of Louisville

Dr. Darid Dunn

Exec VP for Health Affairs

University of Louisville

Dr. Stephen Wheeler, MD, MChE

Associate Dean of Admissions

University of Louisville School of Medicine

Student overview/introduction by members of the planning committee:

Francesca Kingery - Laura Mucenski- Shelia Campbell \& Ben Werner

Kornhauser Auditorium

8:40 am- 10:00 am- Cultural Reflection "Spirituality in an African American Baptist Church" performed by members of Saint Stephens Church, Louisville Kentucky

Kornhauser Auditorium

10:15 am-11:15 am -Concurrent Sessions I

11:15 am-12:00 pm-Lunch \& Poster Sessions

(Provided by Penn Station East Coast Subs)

Kornhauser Library Auditorium

12:00 pm -1:00 pm - Substance Abuse in Health-Care

Chelsea Coogan-Bassett, MD

3rd year Pediatric Resident

University of Louisville

Kornhauser Library Auditorium

1:10 pm- 2:10 pm - Concurrent Sessions II

2:20 pm- 2:40 pm - Closing Remarks \&

Prize Give-A-Ways

\section{Concurrent Sessions}

Japanese Acupuncture in Western Society Concurrent Session I-Dental School Room 131 Concurrent Session II-Medical School Instructional Building B, Room 102

Mark McDonald, MD, FAAP

Associate Professor, Pediatric

University of Louisville

Facilitated by:

Julie Drury, BS, RDH, CHPE

University of Louisville, School of Dentistry

Mennonite Culture and Health-Care

Provisions

Nursing School, $K$-wing Room 2006

Eric \& Leah Kraly

Old Order Mennonite Community

Facilitated by:

Lucy Juett, Center Director, South Central

KY AHEC

Worling Effectively with LGBT Patients

Concurrent Session I -Dental School Rm: 124

Concurrent Session II-Dental School Rm: 131

Lisa Gunterman, BA

Program Coordinator LGBT Services, UofL

Stacie Steinbock, MEd

Sexual Health Advisor

Office of Health Promotion, UofL

Facilitated by:

David Scott, PhD

University of Louisville, School of Dentistry

Michael Rowland, PhD

Office of Medical Education

University of Louisville, School of Medicine

Rebecca Tamas, MD

Department of Psychiatry

University of Louiswille, School of Medicine
Aspects of Practical Differences between Islamic and Western Medicine

Medical School Instructional Building B

Room 202

Hassan M. Hussien Qazzaz, PhD

Senior Research Associate

University of Louisville

Facilitated by: Katie Leslie, MS

UofL Minority \& Rural Affairs

Applying Military Cultural Competencr to the Care of Veterans/Service Members Kornhauser Library Auditorium

Charlie Meador, Chief Warrant Officer, US Army Reserves

Thao Tran, NorthWest AHEC Clinical Coordinator Jonathan "Kelly" Marcum, CSW, Outreach Tech, Louisville Veterans Center

Interlink Counseling Services, Color Guard

Facilitated by:

Dr. Lee Mayer, DMD

University of Louisville, School of Dentistry

Child Discipline \&: Maltreatment: How Do We Define Abuse \& Neglect in a Multicultural Society?

Baxter I Research Bldg, Basement Auditorium

Melissa Currie, MD, FAAP

Associate Professor and Dir. Forensic Medicine University of Louisville, School of Medicine

Facilitated by:

Dr. Deborah Davis, PhD

Professor of Pediatrics, Dir. Diversity Committee University of Louisville, School of Medicine

Students \& Their View-Points on Culture Dental School, Room 119, 119.4,119B

Facilitated by:

Francesca Kingery-Med School Class of 2016 Laura Mucenski-Med School Class of 2015 Sheila Campbell- Dental Hygiene Class of 2013 Ben Werner-Dental School Class of 2014

\section{FIGURE 1: 2012 Cultural Competency Workshop at the University of Louisville.}




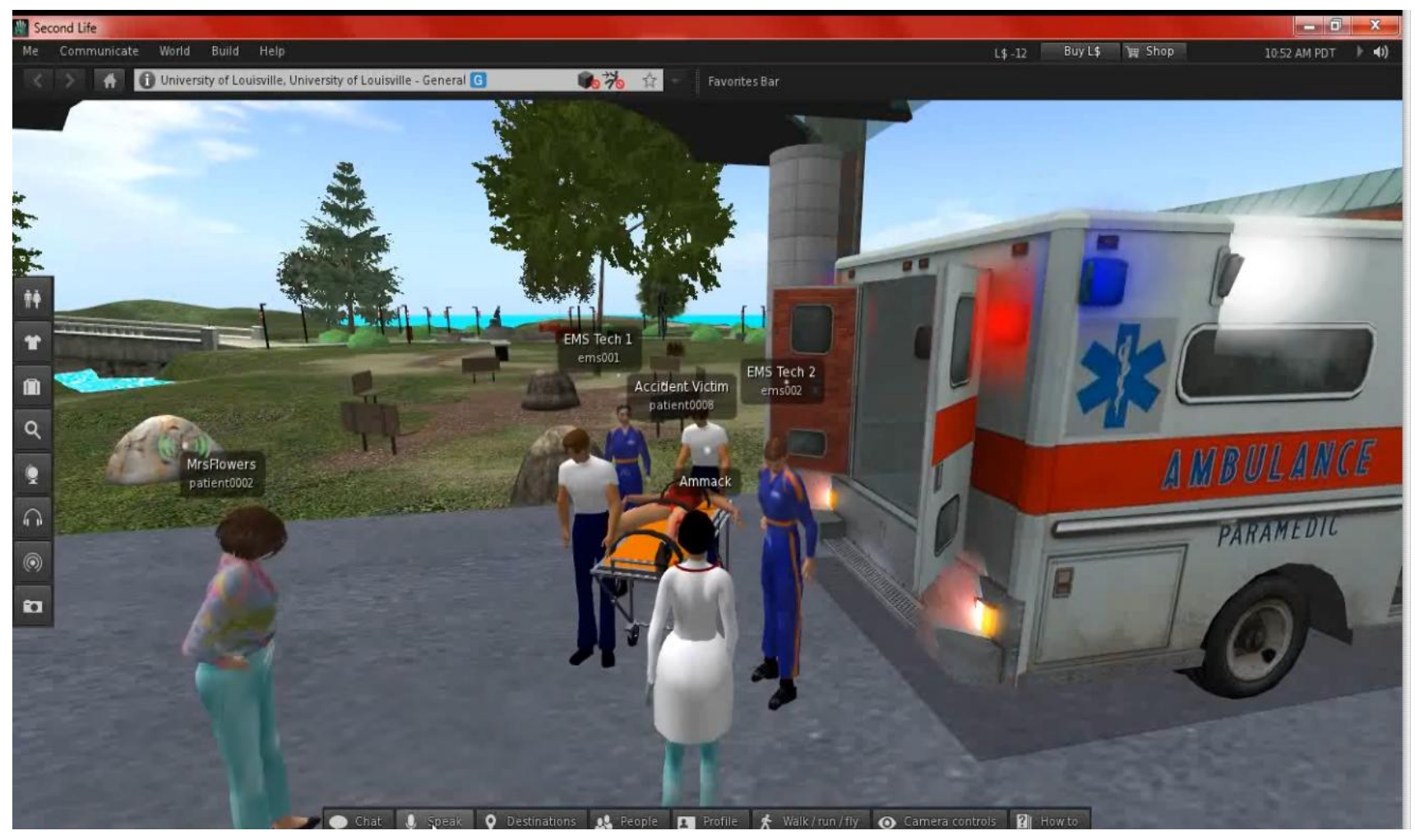

FIGURE 2: This screen shot from Second Life ${ }^{\mathrm{TM}}$ depicts the hospital scenario setting. The transgendered patient has just arrived to the emergency room without receiving any treatment from the emergency medical service (EMS) workers. 


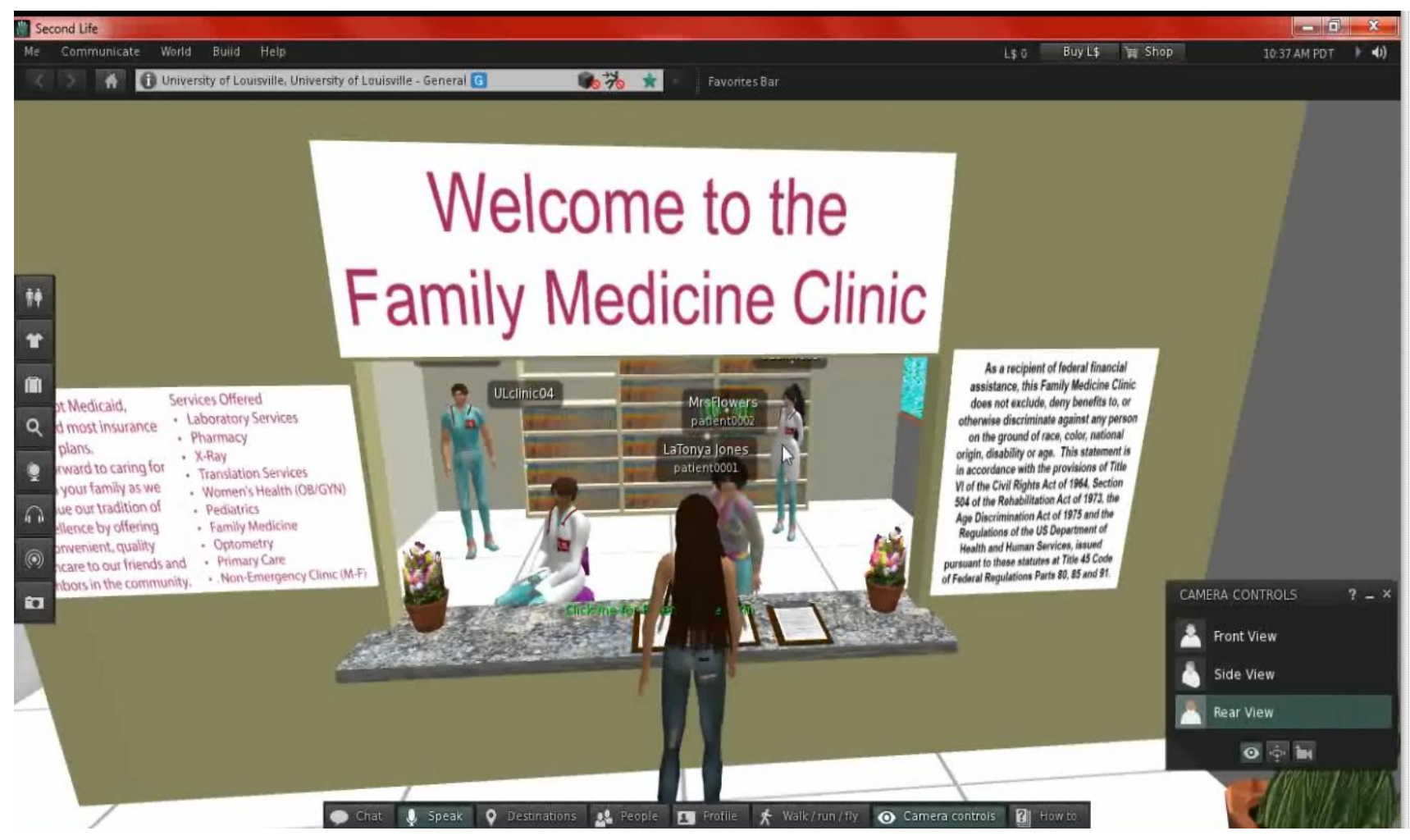

Figure 3: This screen shot from Second Life ${ }^{\mathrm{TM}}$ depicts lesbian patient, LaTonya, checking in at the registration desk in our Second Life ${ }^{\mathrm{TM}}$ doctor's office. 


\section{Second Life ${ }^{\mathrm{TM}}$ USER GUIDE}

Getting Started:

Click JOIN NOW

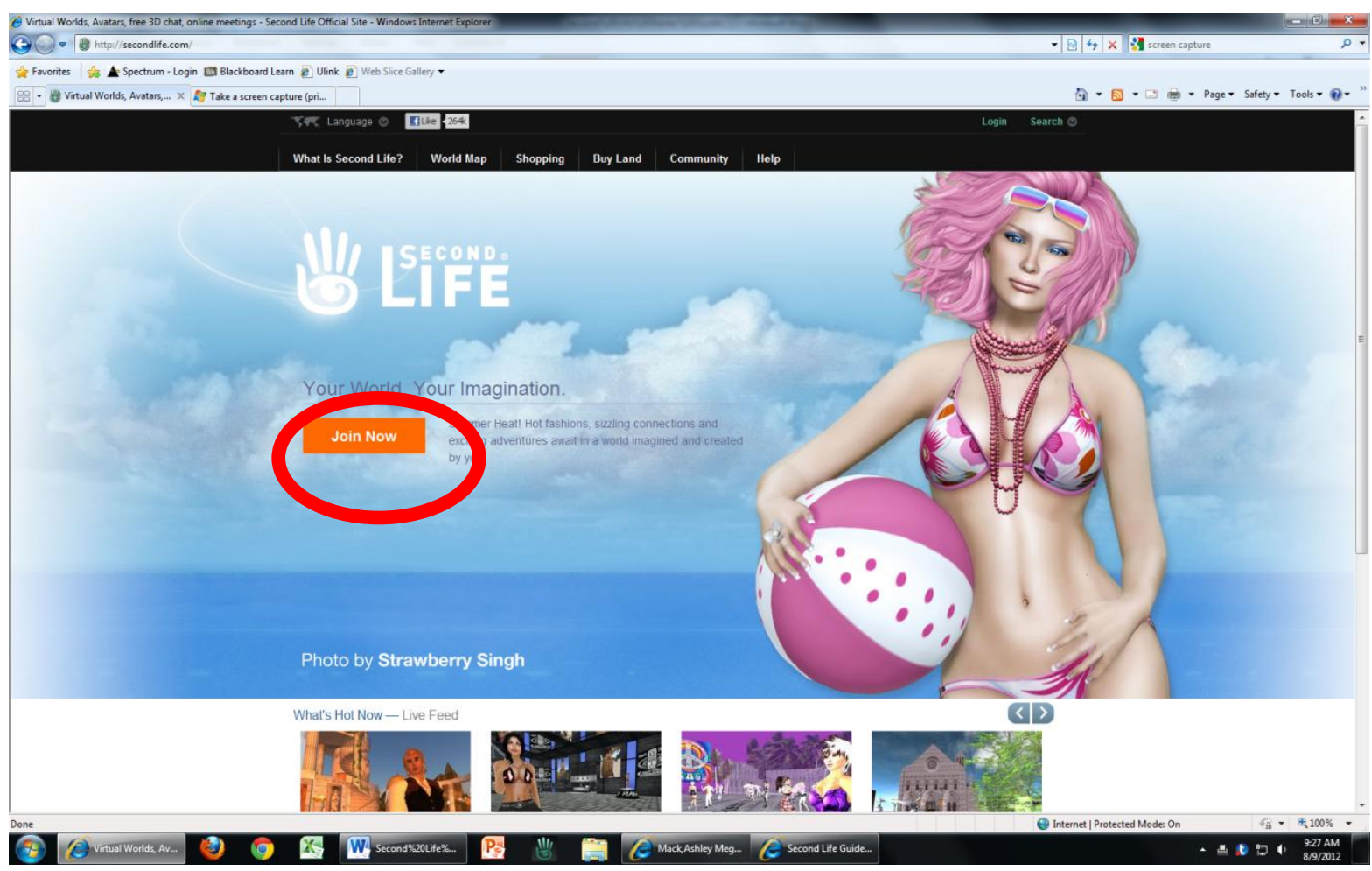

FIGURE 4: User guide developed for volunteer that played the roles of our characters in each scenario. The User Guide will continue over the next several pages (Pages 89-102) 
SECOND LIFETM GUIDE PAGE 2

Choose THIS AVATAR (Avatar you will initially appear as in Second Life $^{\mathrm{TM}}$ )

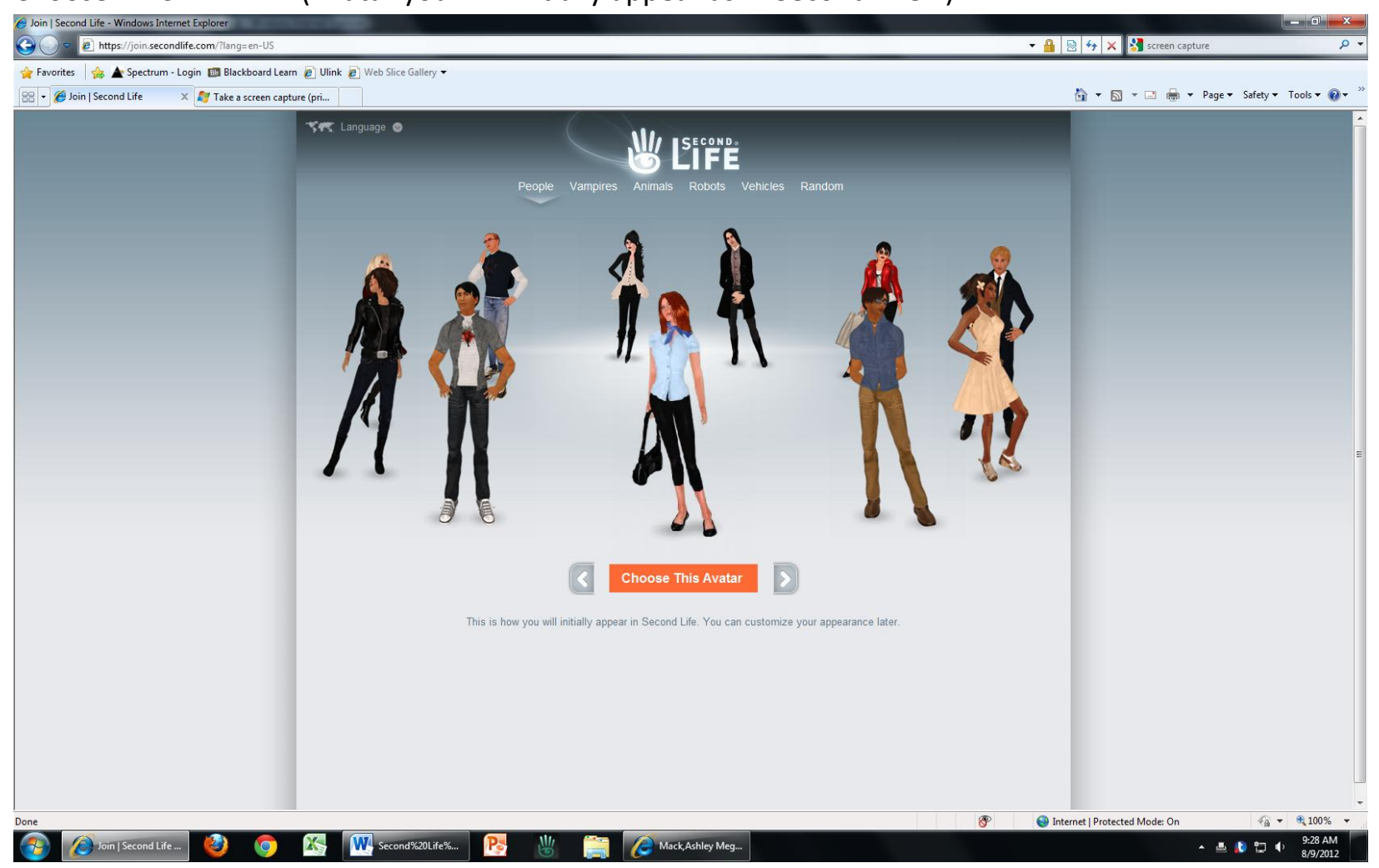




\section{SECOND LIFETM GUIDE PAGE 3}

Choose USERNAME (It will check for availability. If username already taken, you will need to choose another username)

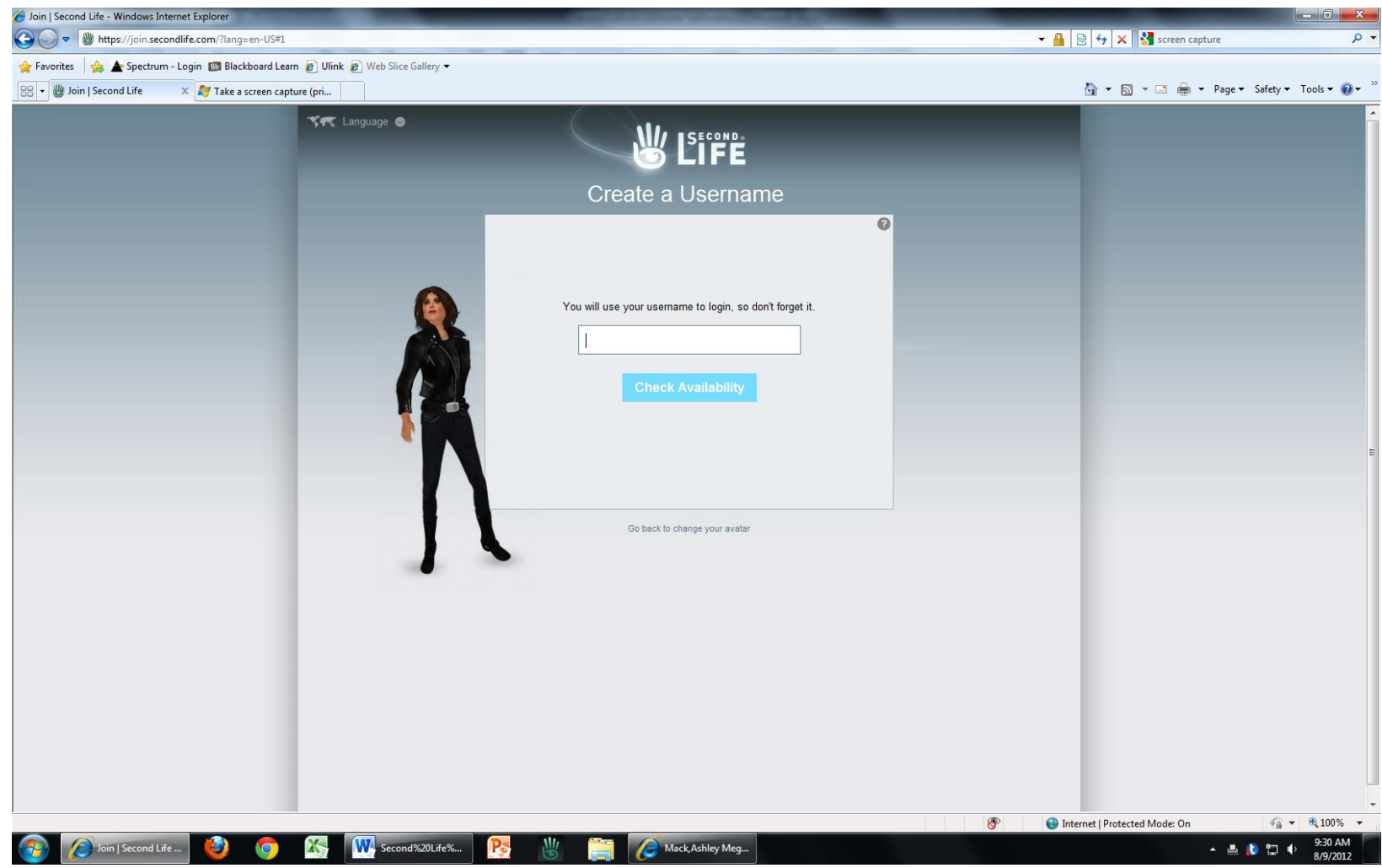

Fill in: Email, Date of Birth, Password, Security Question, and Security Answer 


\section{SECOND LIFE ${ }^{\text {TM }}$ GUIDE PAGE 4}

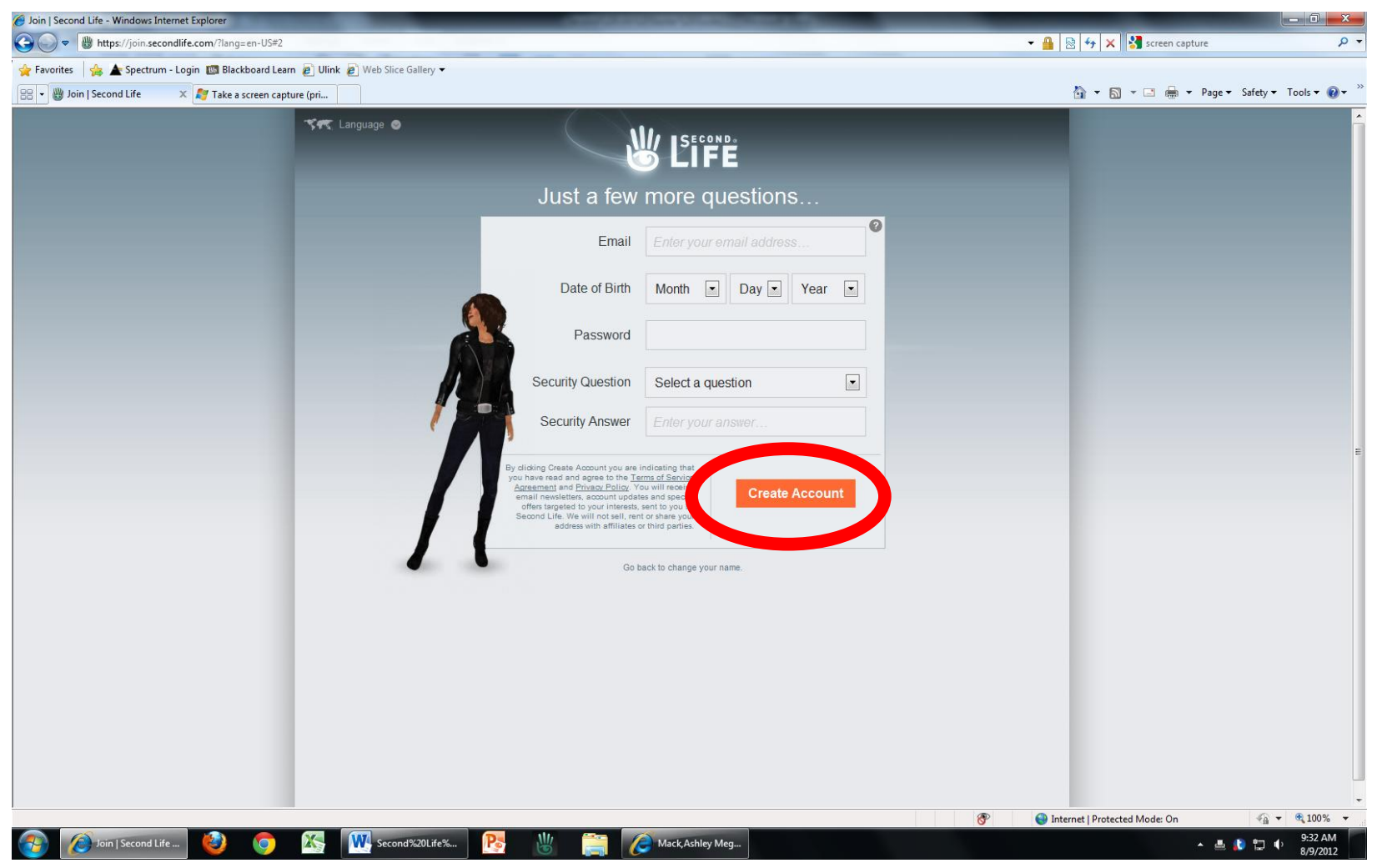

Click: Create Account

Choose Free or Premium Account 


\section{SECOND LIFETM GUIDE PAGE 5}

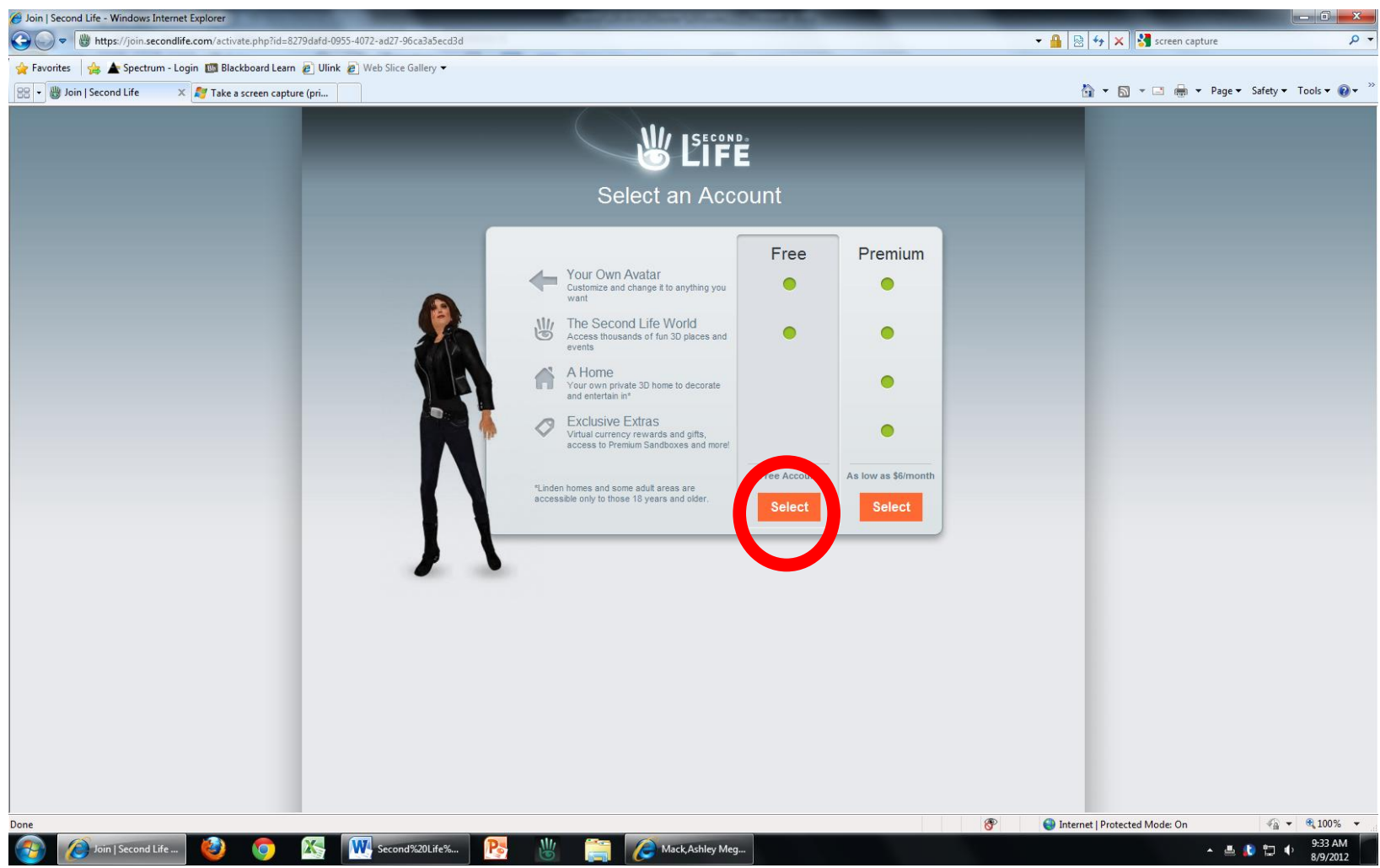

Click Download and Install Second Life ${ }^{\mathrm{TM}}$ (This may take a few minutes) 
SECOND LIFE ${ }^{\mathrm{TM}}$ GUIDE PAGE 6

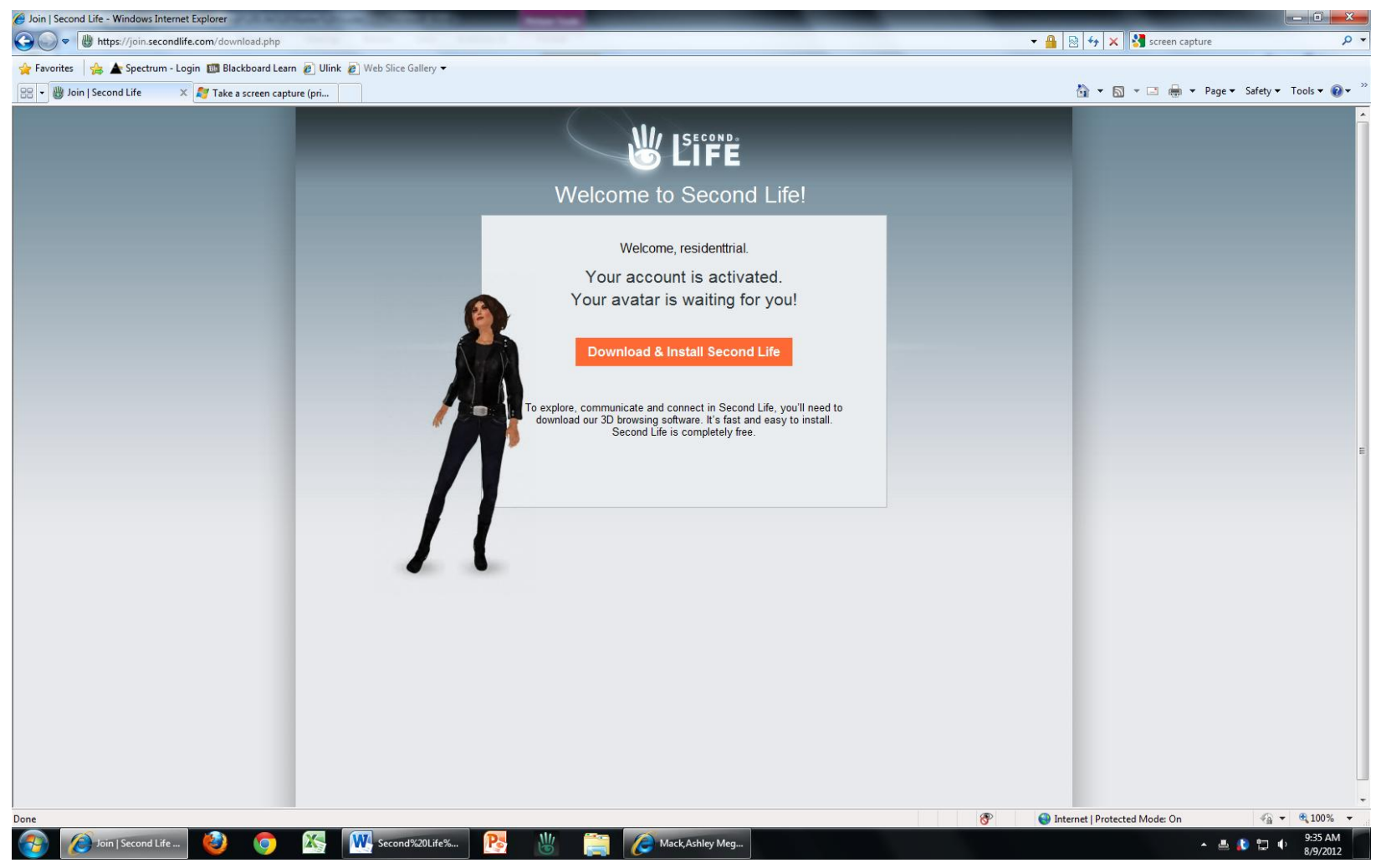




\section{SECOND LIFE'M GUIDE PAGE 7}

A box may appear asking you if you would like to update your graphics clip. You can EXIT OUT of this box.

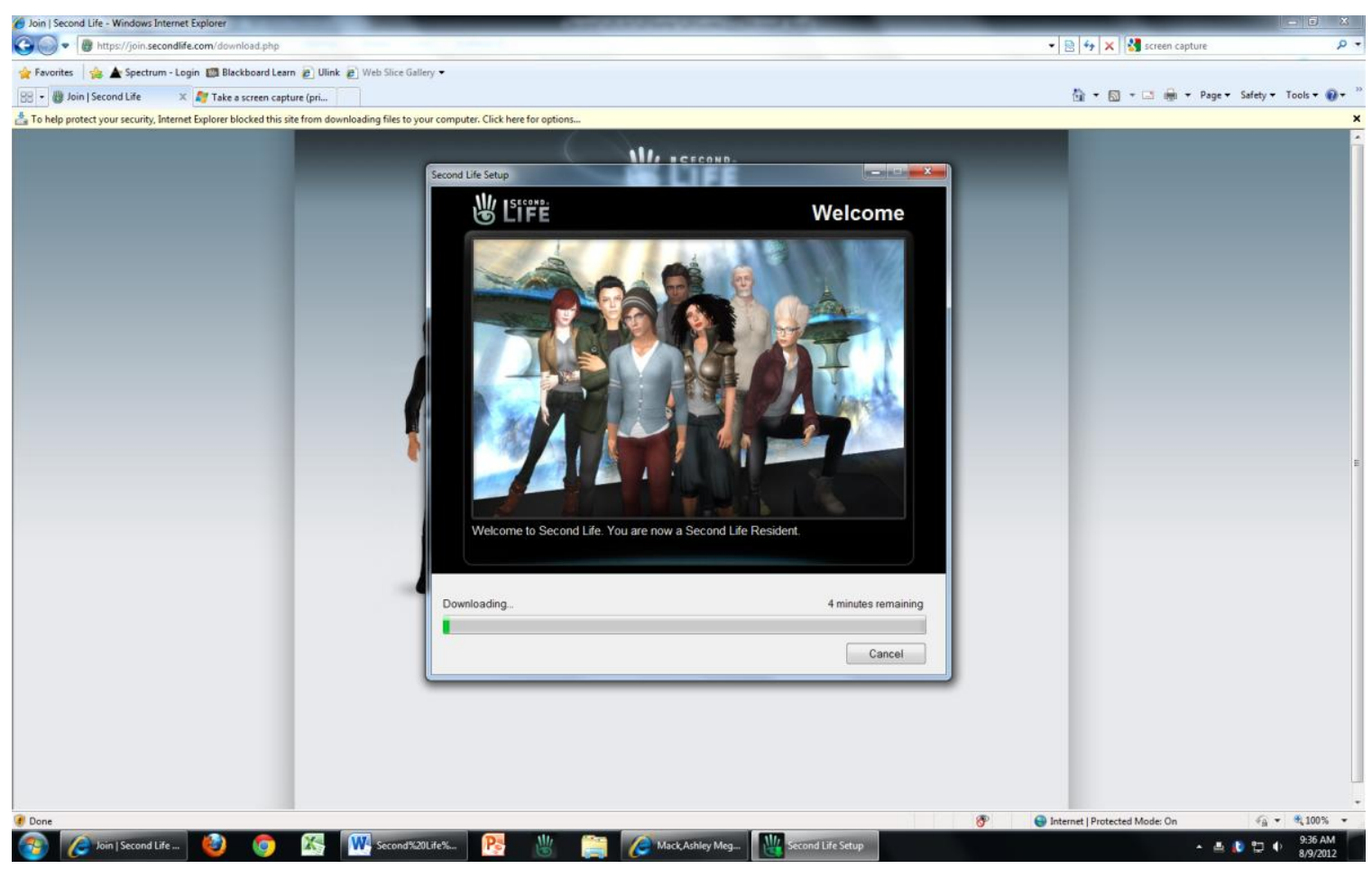




\section{SECOND LIFETM GUIDE PAGE 8}

Terms of Agreement will Load. Click the I agree to the Terms of Service and Privacy Policy. On the bottom control panel, on the far right, there is a How To Button you can Click that will Review Basic Controls with you.

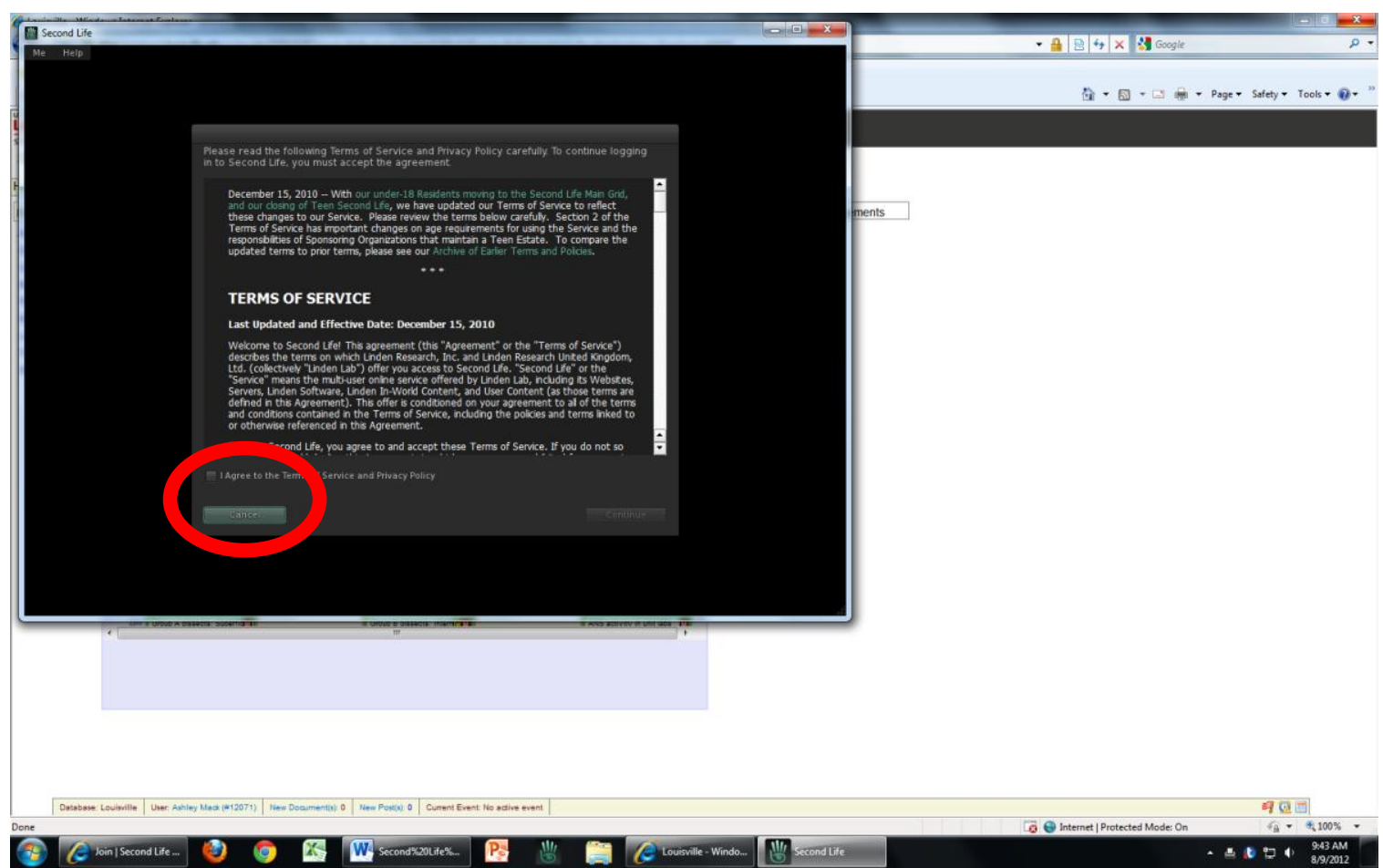


SECOND LIFE ${ }^{\mathrm{TM}}$ GUIDE PAGE 9

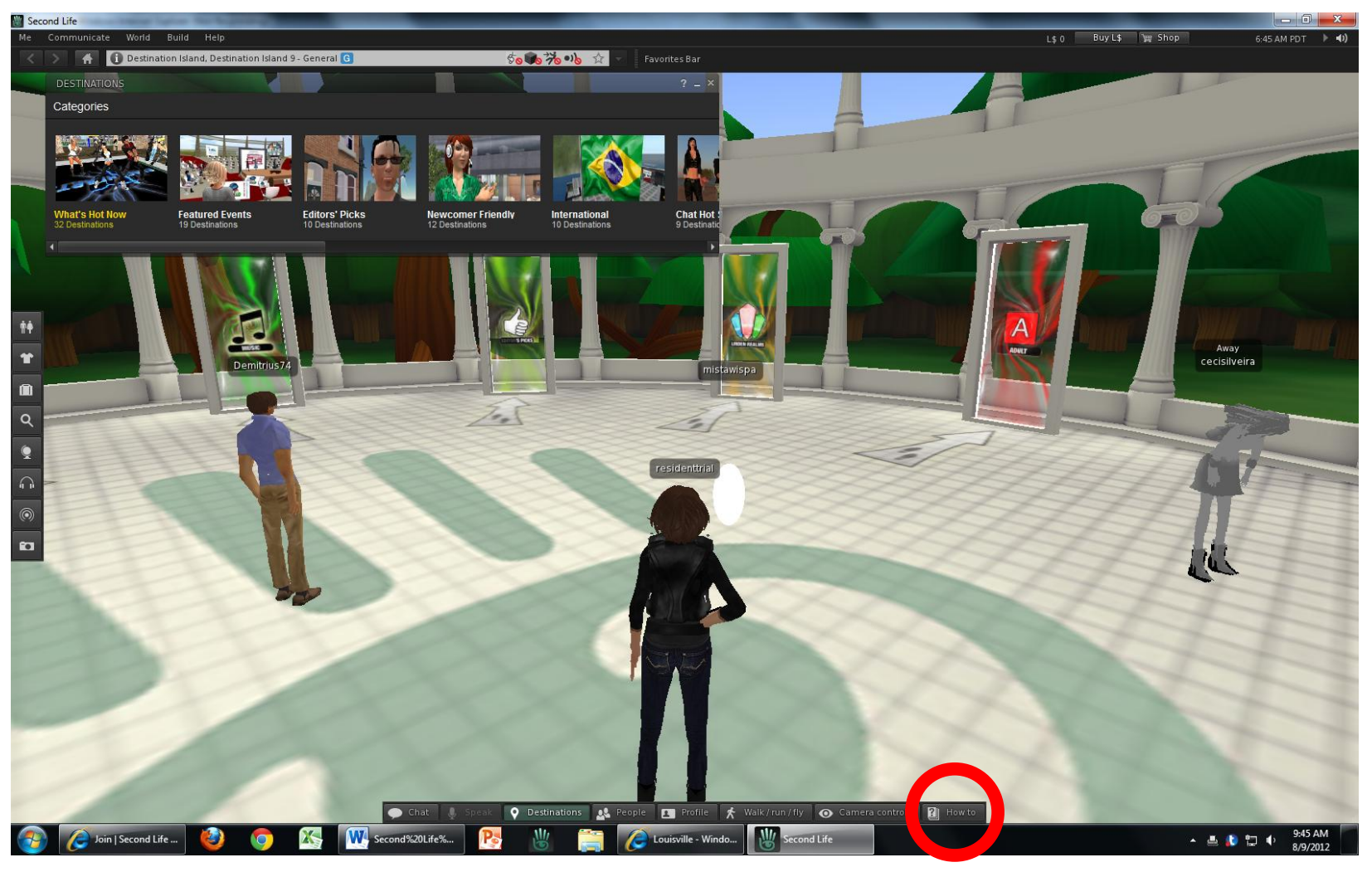




\section{SECOND LIF ${ }^{\mathrm{TM}}$ GUIDE PAGE 10}

The How To Guide will walk you through Basic Control:

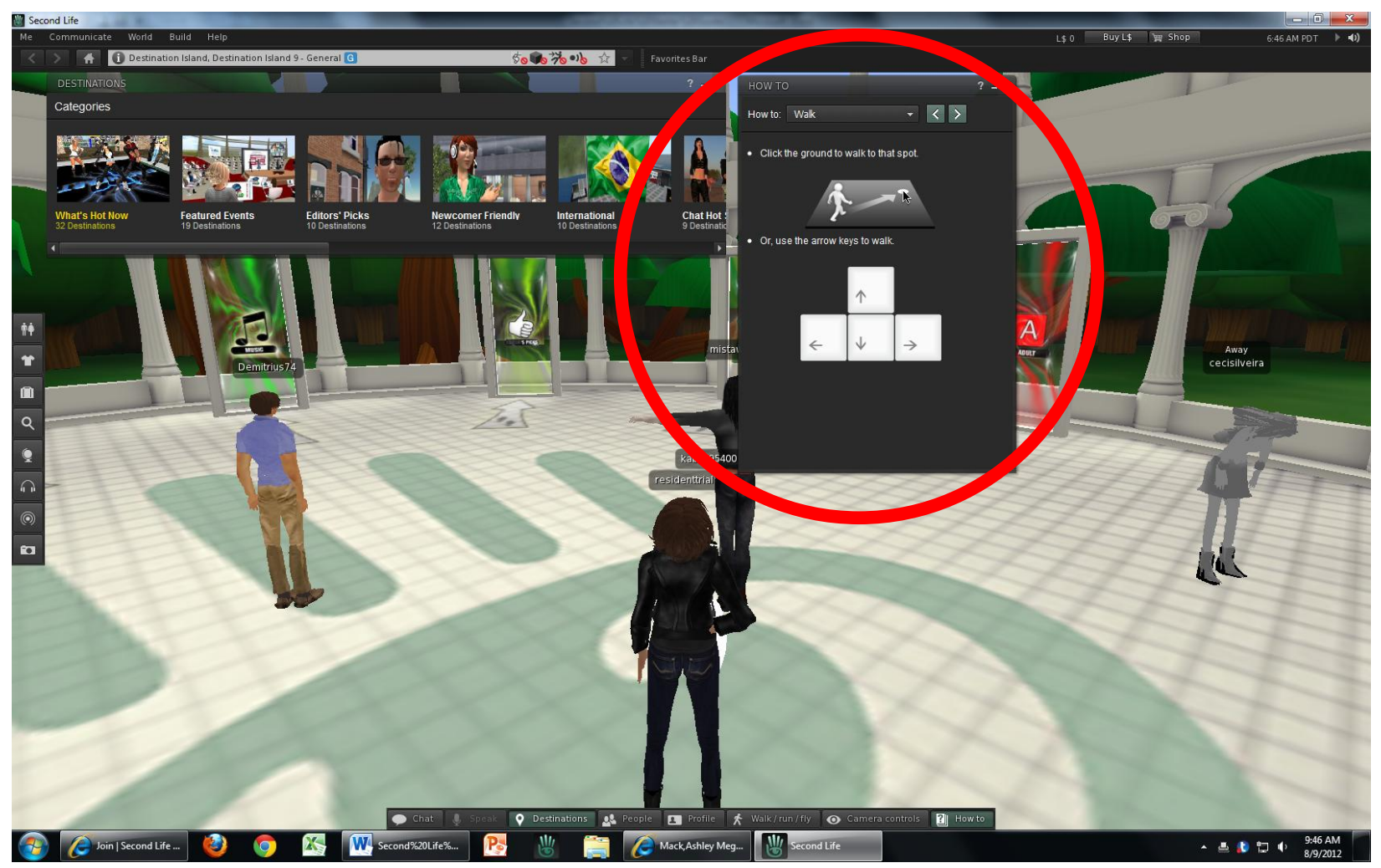

\section{Basic Controls:}

\section{Change PROFILE:}

Click the Edit Button and you can Change Current Information and add Interests

\section{Change AVATAR:}

Click on the avatar button. You may select a different avatar.

Two ways to WALK:

- Click on the ground the spot you want to walk to

- Use the arrow keys to walk around. 


\section{SECOND LIFE ${ }^{\mathrm{TM}}$ GUIDE PAGE 11}

How to FLY:

Click PAGE UP to fly and PAGE DOWN to land. Also, you can select E to fly and C to land.

Interact with an OBJECT:

Right-click an object to interact with it. Select an option from the menu. There are objects you can sit on, buy, dance, and more.

To focus on an object click ALT + Left-click .

\section{CHAT:}

Click the Chat window. Type a message and click return. Private chats are also available.

Change VIEW:

Click View at Bottom of Screen and rotate your view.

Or you can rotate the camera by holding the ALT + left-click and rotate up or down and side to side (to zoom)

Change DESTINATION:

Click Destinations to change Destination.

After scrolling through the Basic Controls you will want to go to one of the orientation islands. Choose Destinations from the bottom menu. 


\section{SECOND LIFE ${ }^{\mathrm{TM}}$ GUIDE PAGE 12}

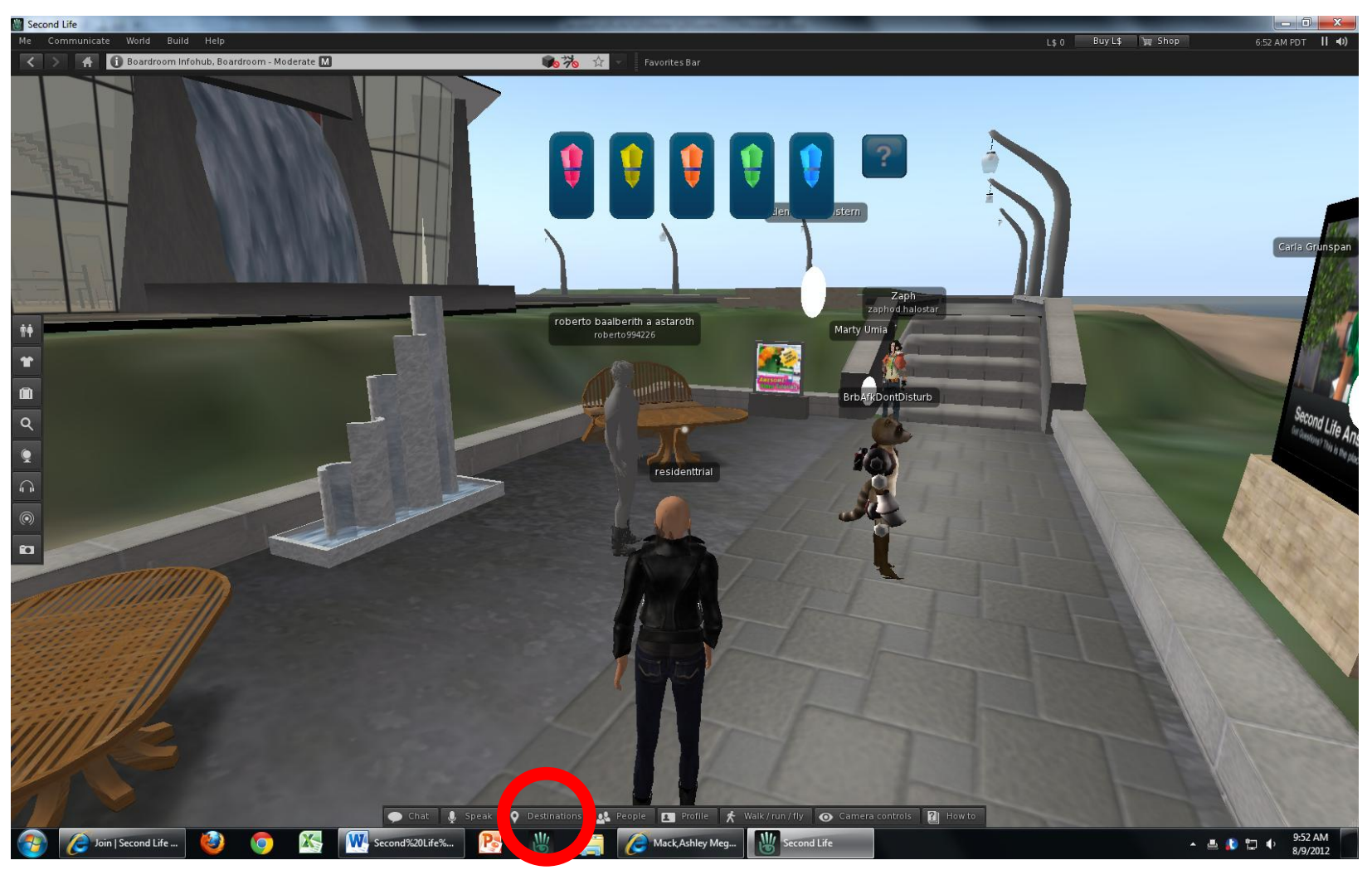




\section{SECOND LIFE ${ }^{\mathrm{TM}}$ GUIDE PAGE 13}

Click on Newcomer Friendly

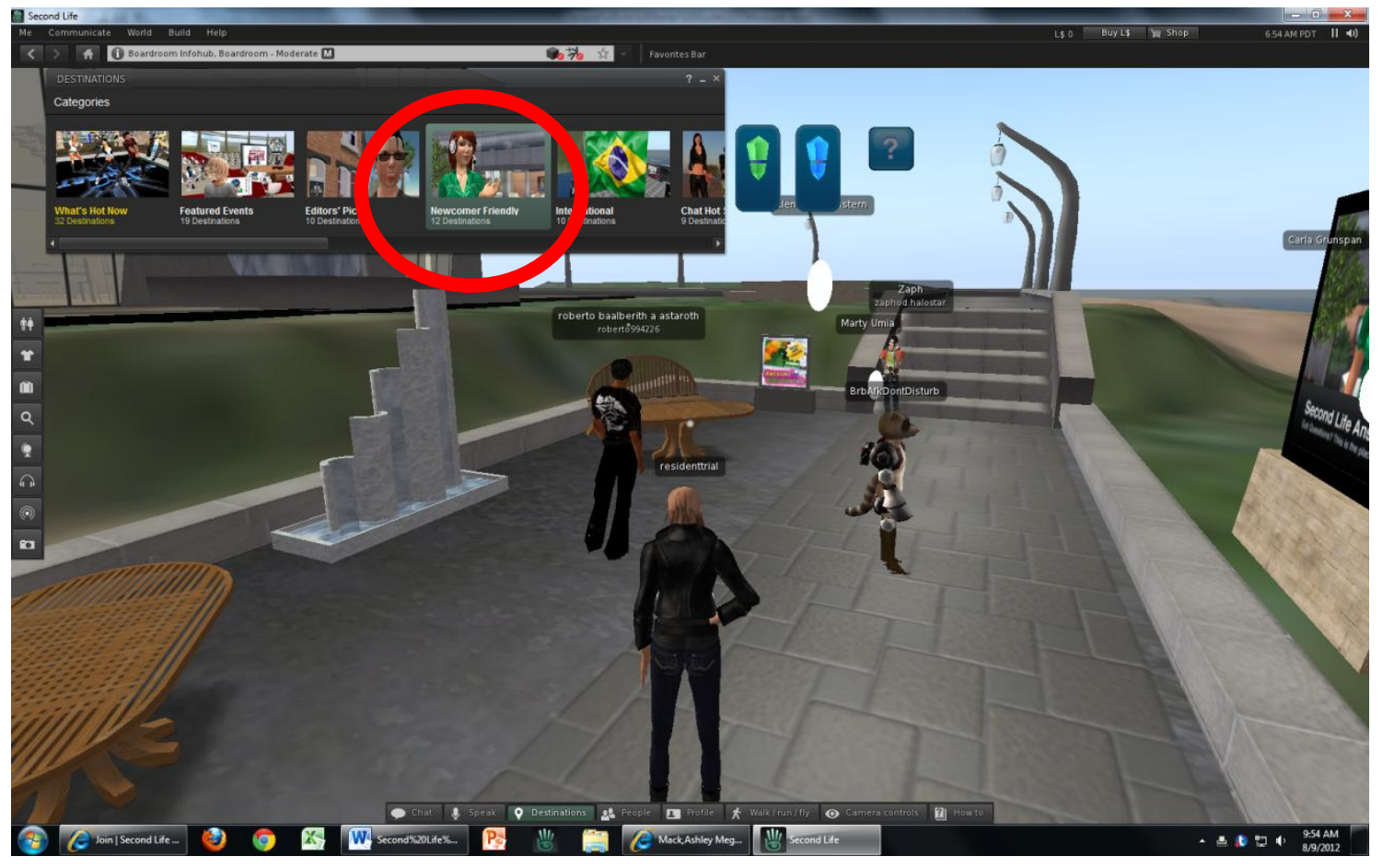




\section{SECOND LIFE ${ }^{\mathrm{TM}}$ GUIDE PAGE 14}

Try New Citizens Incorporated. It will guide you into all the controls of Second Life ${ }^{\mathrm{TM}}$.

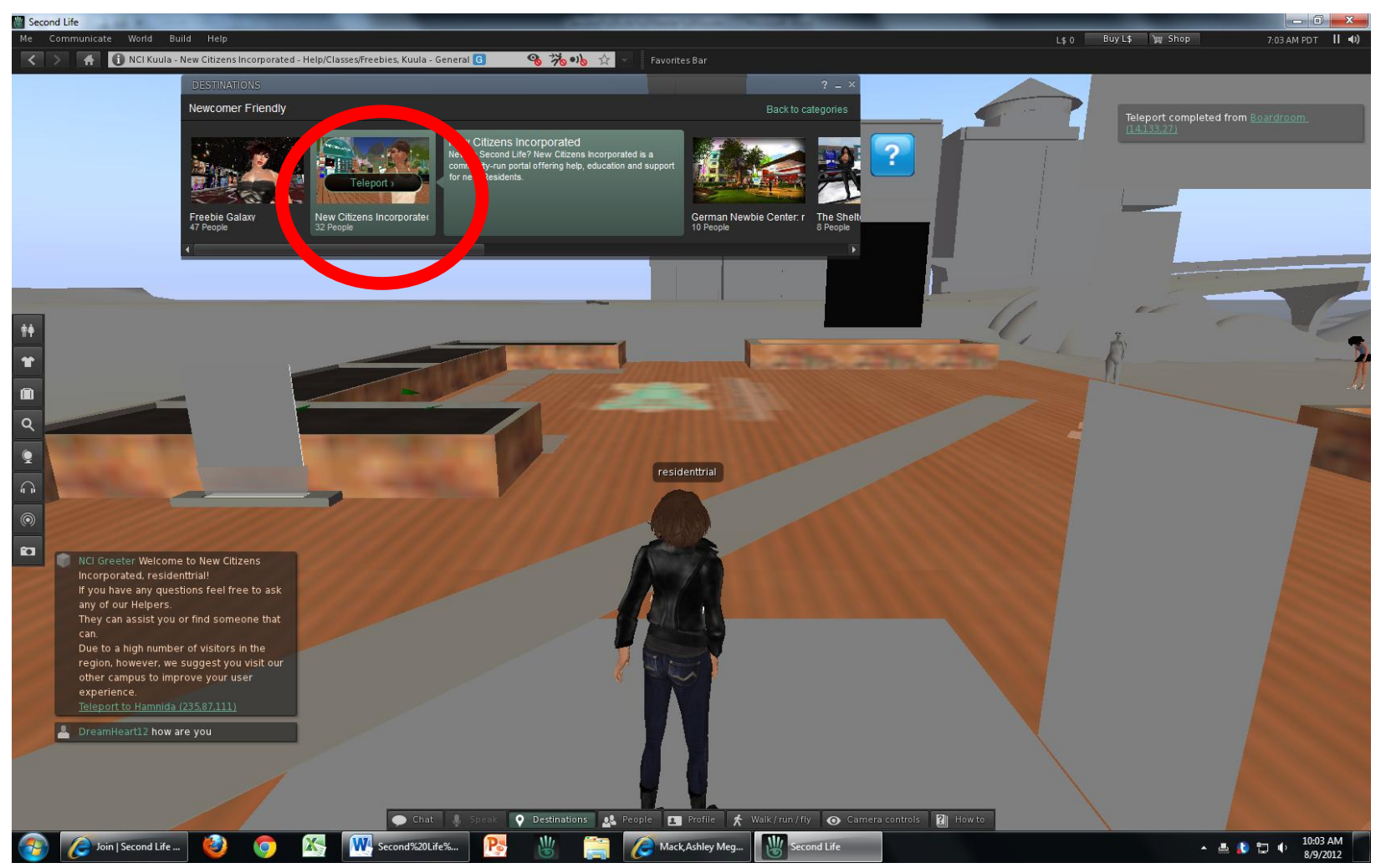

FIGURE 4: User guide developed for volunteer that played the roles of our characters in each scenario. 


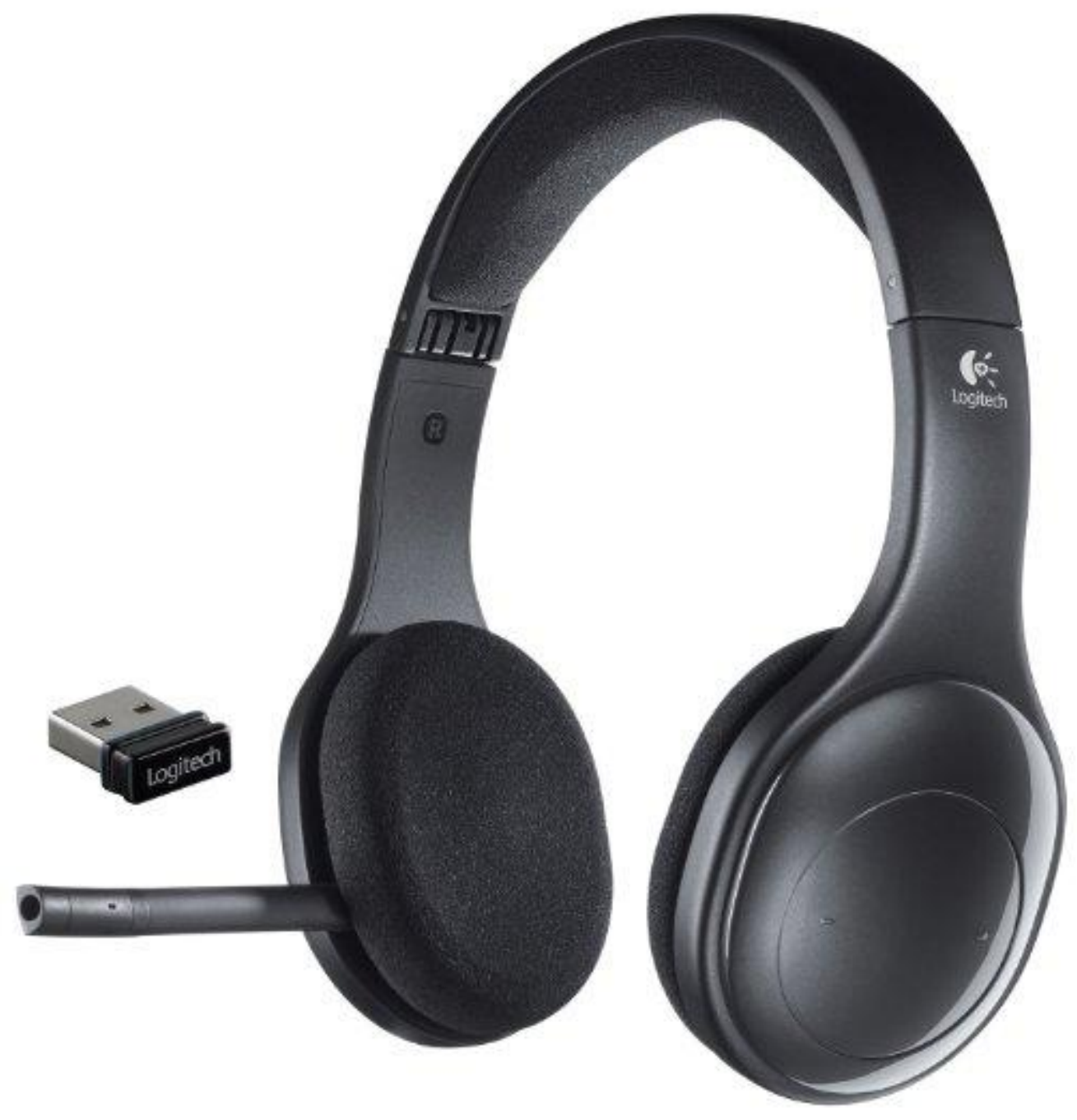

FIGURE 5: Logitech Headphones used to hear and speak in Second Life ${ }^{\mathrm{TM}}$ during the scenarios. 
2012 LEBT Workshop Survey

1. Please pook podec, the following components of this acssion from (1) most effective to (4) least effective.

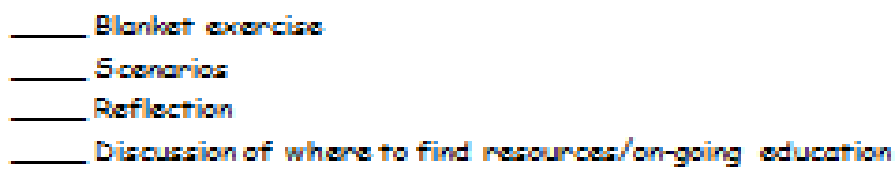

2. On a scale from 1 (being poor) and 6 (being excellent) did this acssion enhance your knowlodge of LGBTQ identities and community?

$$
\begin{array}{lllll}
1 \text { Poor } & \overline{3} & \overline{3} & \overline{5 \text { Excellent }}
\end{array}
$$

3. On a scale from 1 (being poor) and 6 (being excellent) did this session increase your awareness of disparate health problems with in this population?

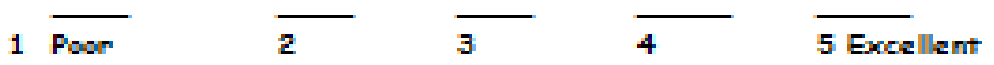

4. On a scale from 1 (being poor) and 6 (being excellent) did this session enhance your knowledge of barriers to healthcare specific to this population?
$1 \longdiv { \text { Poor } }$

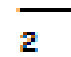
3
4
5 Excellent

6. On a scale from 1 (being poor) and 6 (being excellent) did this session increase your sensitivity and compassion for LGBTQ patients?
$1 \longdiv { \text { Poor } }$
$\overline{2}$
3
4
5 Excellent

6. On a scale from 1 (being poor) and 6 (being excellent) How would you rate this session overall?
1 Poor
2
3
4
5 Excellent

7. After this presentation, do you foel more confident working with this population? Yes No Uncertain

FIGURE 6: Post-survey questionnaire given to each of the participants in both the Second Life ${ }^{\mathrm{TM}}$ and Traditional workshop setting. 
8. Did you self-select the LGBT session or were you placed in this acssion becouse the other sessions were alnoody full? I self-eclactod this acssion

I was placed in this acssion becouse the other sessions were alneody full

9. Overall, I was very engoged by this preaentation

1 Strongly disagree $\quad 2 \quad 3 \quad 4 \quad 3$ Strongly agree

10. What was the most valuable information you learnod in this session?

11. Do you have any other questions/comments about this population you would be willing to share?

FIGURE 6: Post-survey questionnaire given to each of the participants in both the Second Life ${ }^{\mathrm{TM}}$ and Traditional workshop setting. 
Access to health care is the same for Lesbian, Gay, Bisexual and Transgender (LGBT) persons as for other members of the population.

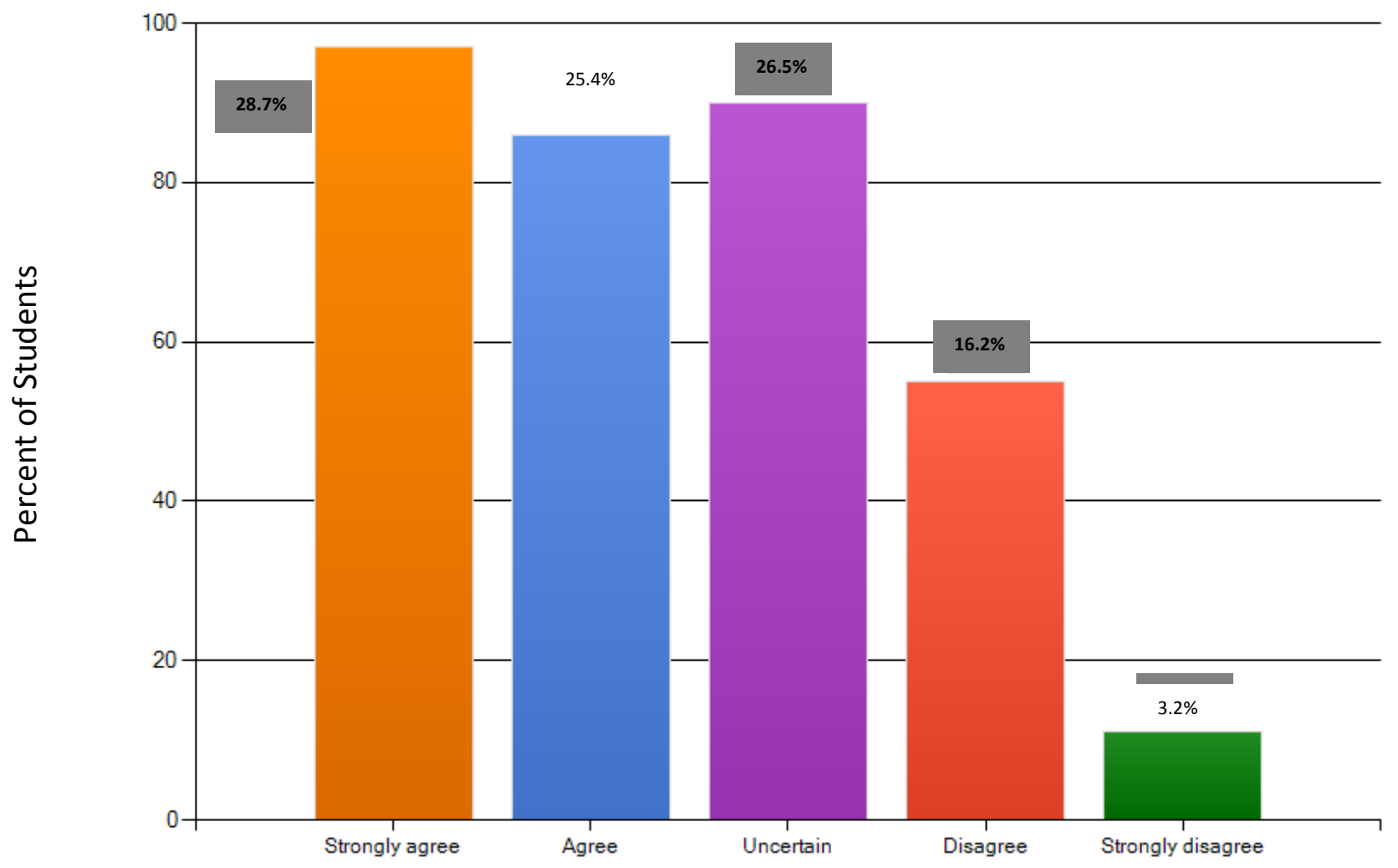

FIGURE 7: Pre-Session Survey Results: Question 5 of the pre-session survey of the 343 students attending the $2012 \mathrm{U}$ of L Cultural Competency Workshop Series. 
Most LGBT young people do their first "coming out" to a nonparent adult (e.g. a teacher or doctor)

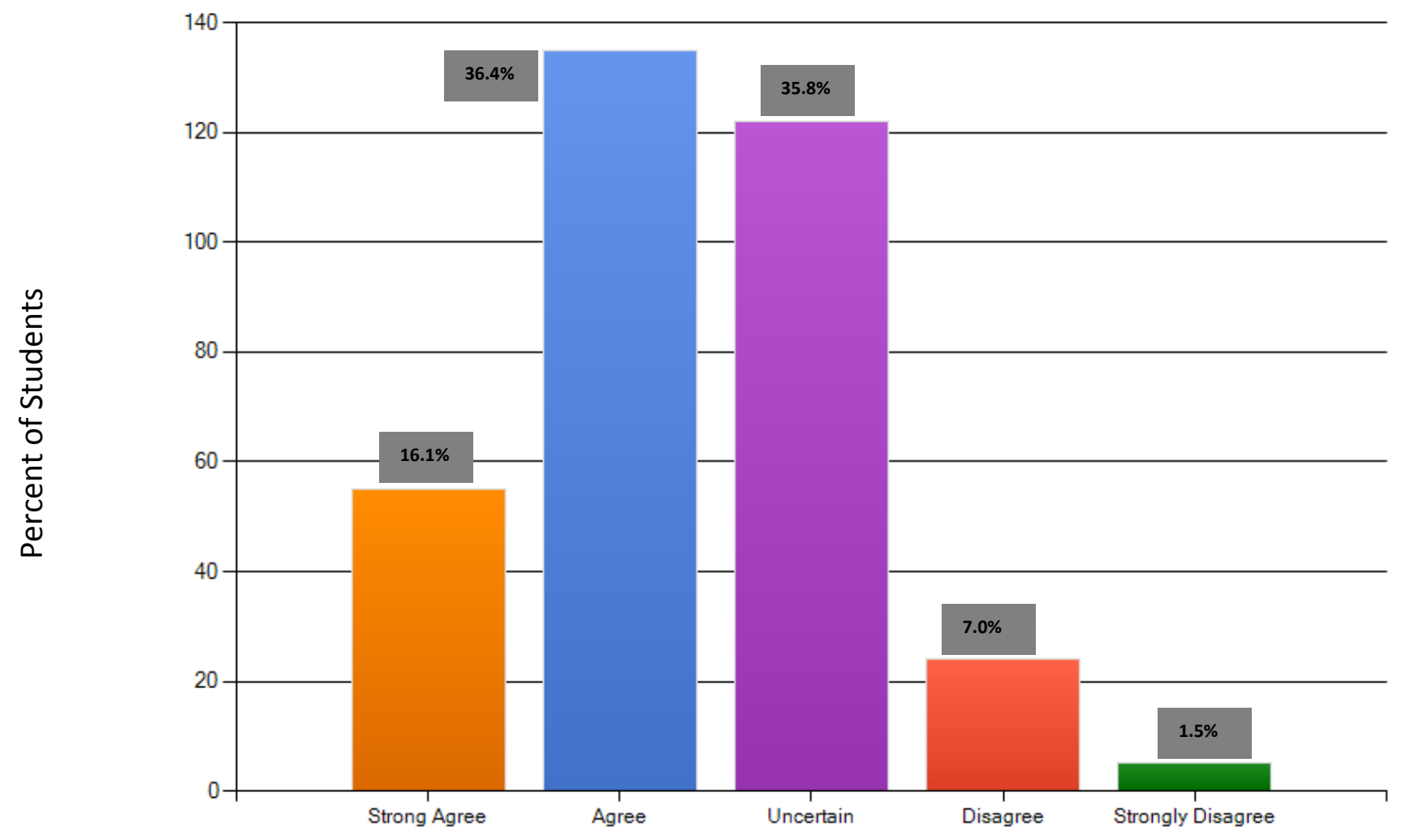

FIGURE 8: Pre-Session Survey Results: Question 8 of the pre-session survey of the 343 students attending the $2012 \mathrm{U}$ of L Cultural Competency Workshop Series. 
LGBT people are less likely than heterosexual people to be in long-term monogamous relationships.

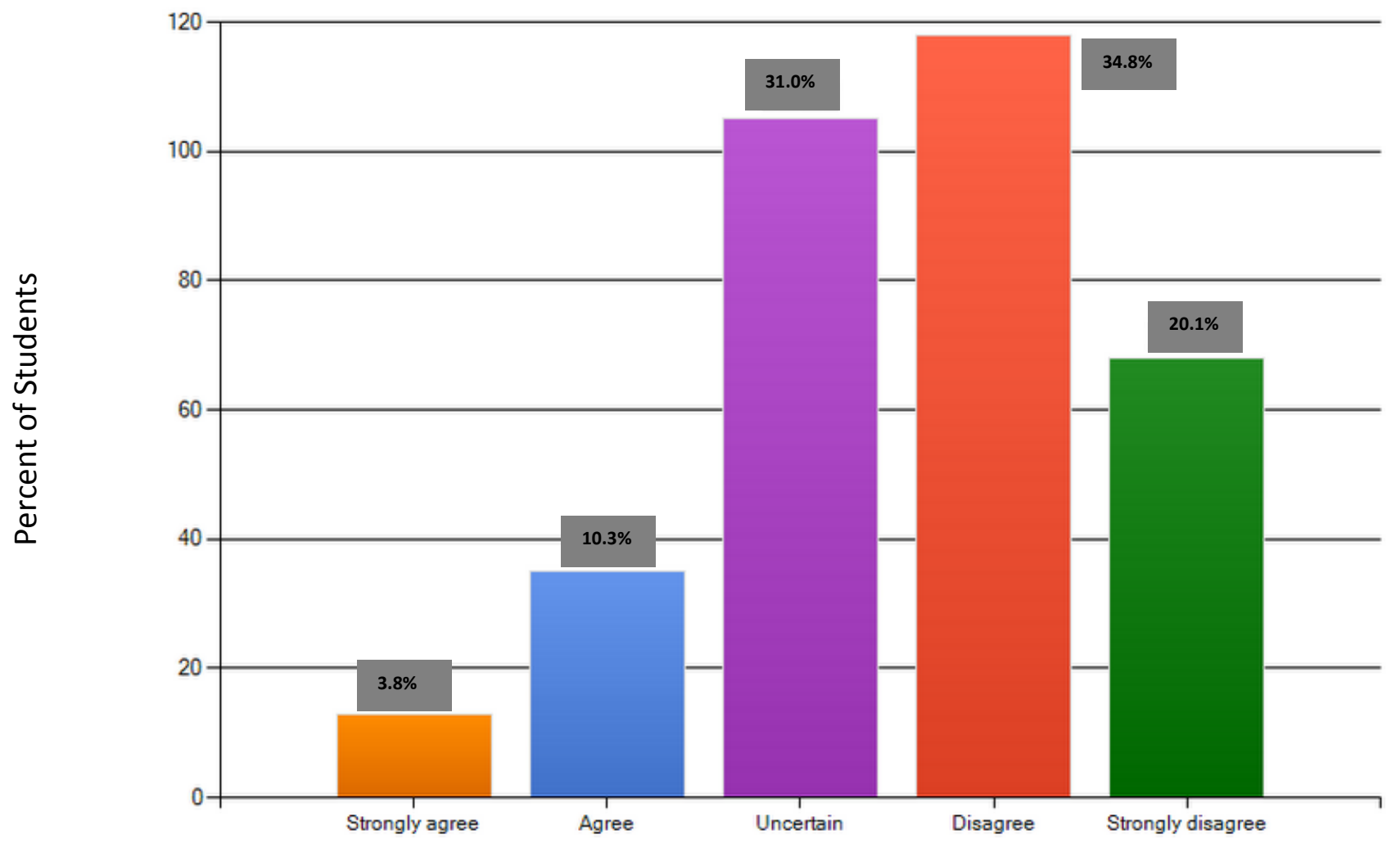

FIGURE 9 Pre-Session Survey Results: Question 11 of the pre-session survey of the 343 students attending the $2012 \mathrm{U}$ of $L$ Cultural Competency Workshop Series. 
LGBT populations have unique health risks and health needs.

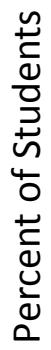

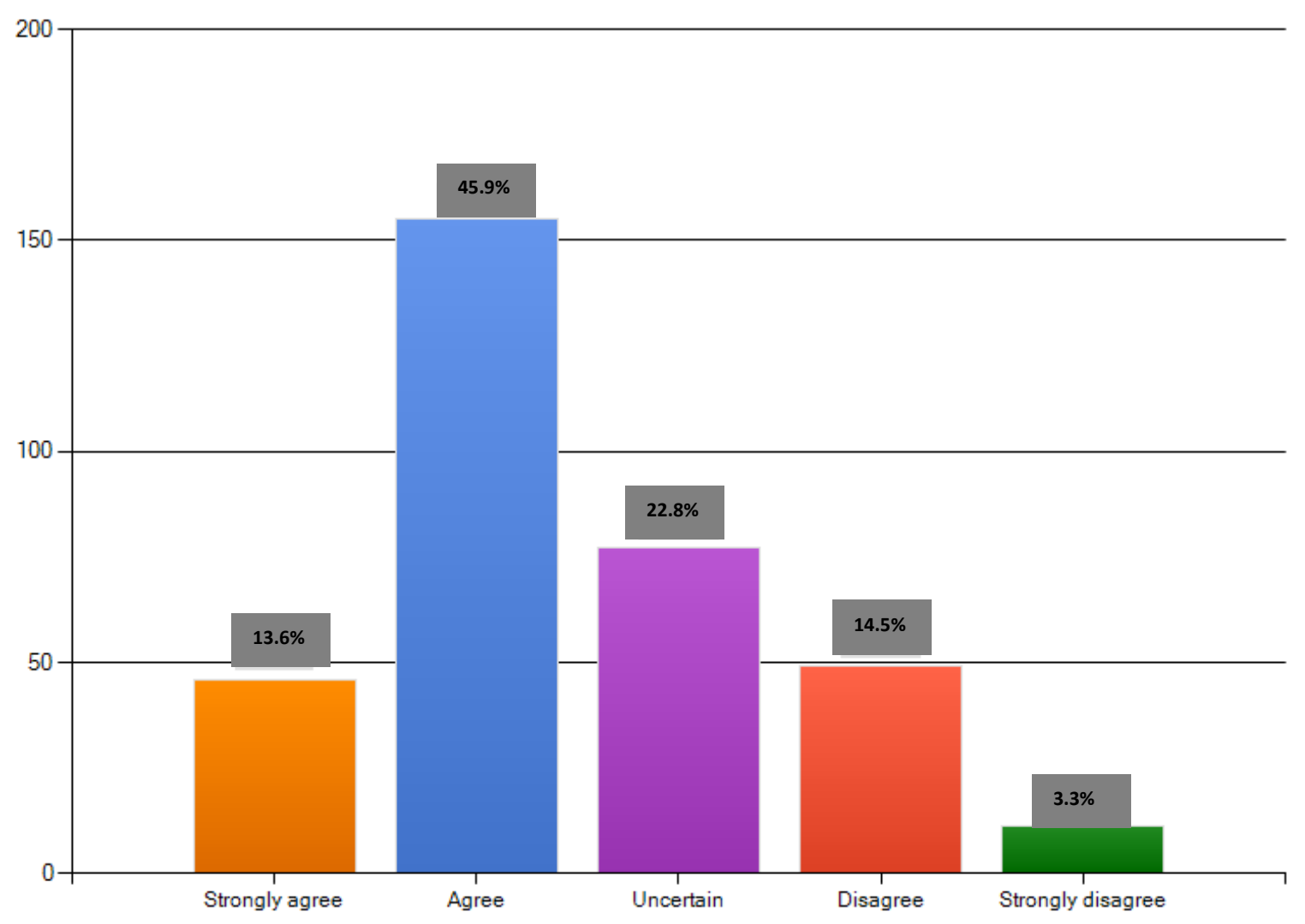

FIGURE 10: Pre-Session Survey Results: Question 16 of the pre-session survey. 
As a health care provider, I feel it is important for me to know about my patients' sexual orientation, sexual practices and gender identity.

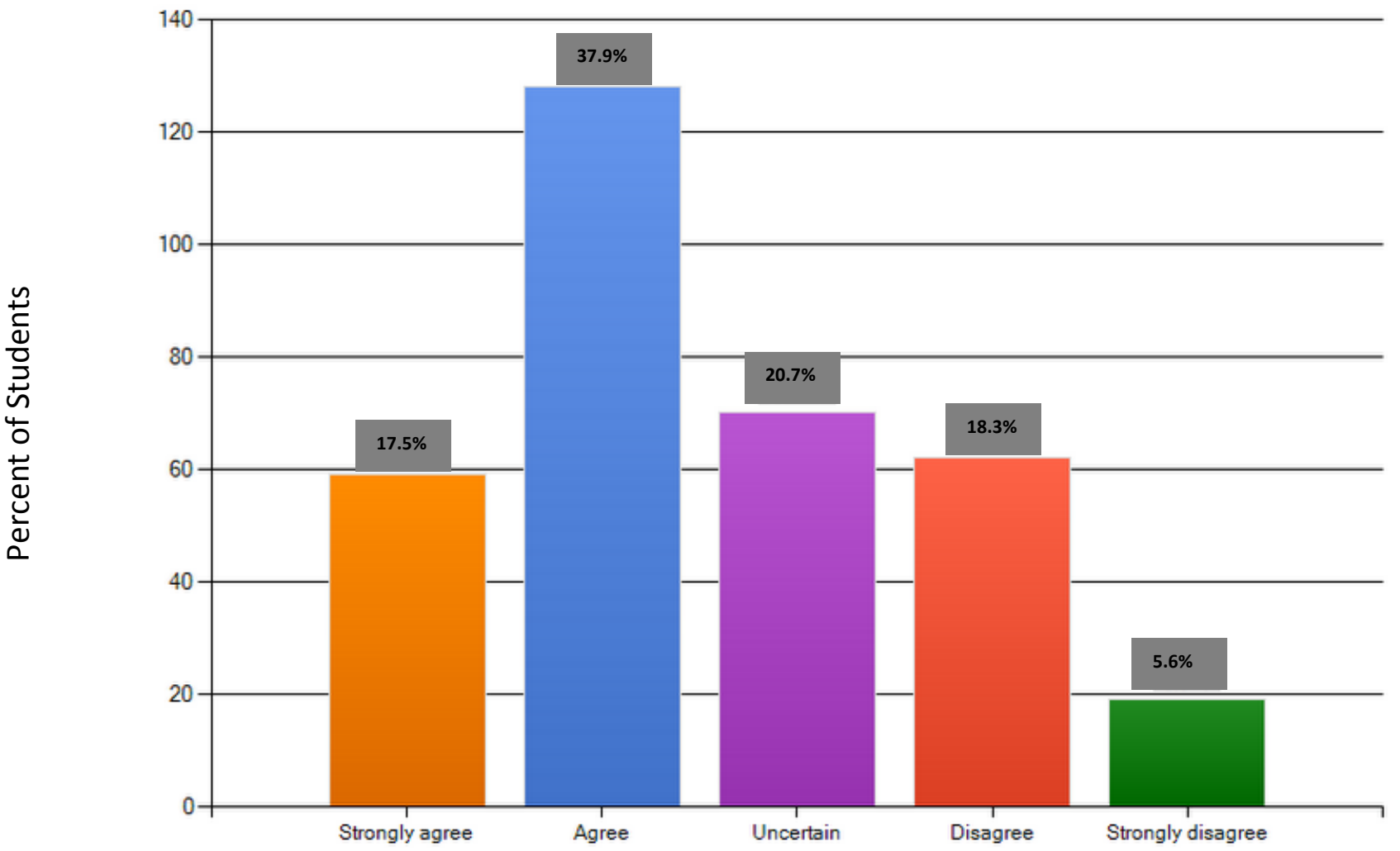

FIGURE 11 Pre-Session Survey Results: Question 21 of the pre-session survey of the 343 students attending the $2012 \mathrm{U}$ of L Cultural Competency Workshop Series. 
I would prefer not to treat patients with gender identity issues.

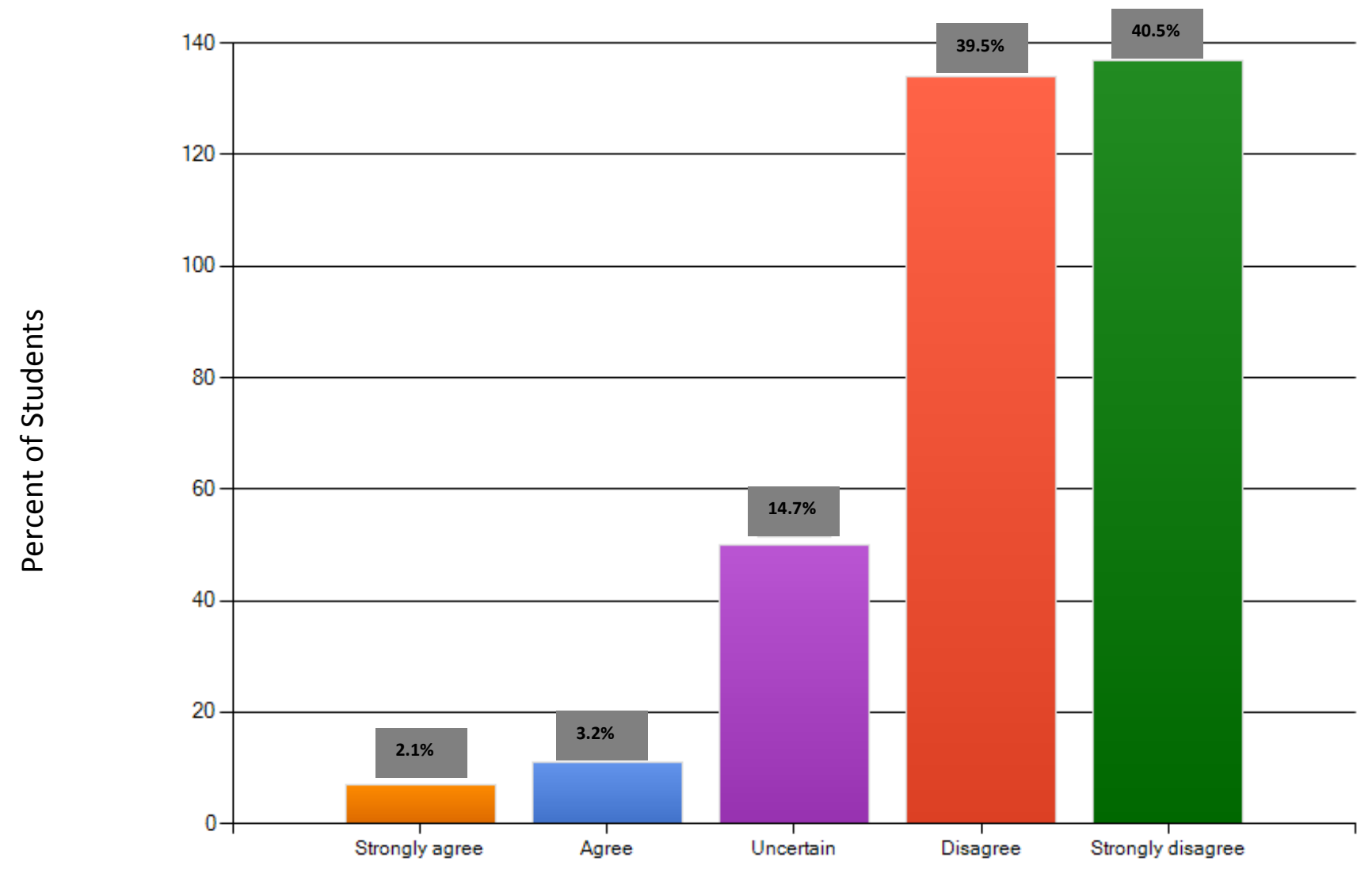

FIGURE 12: Pre-Session Survey Results: Question 26 of the pre-session survey of the 343 students attending the $2012 \mathrm{U}$ of L Cultural Competency Workshop Series. 


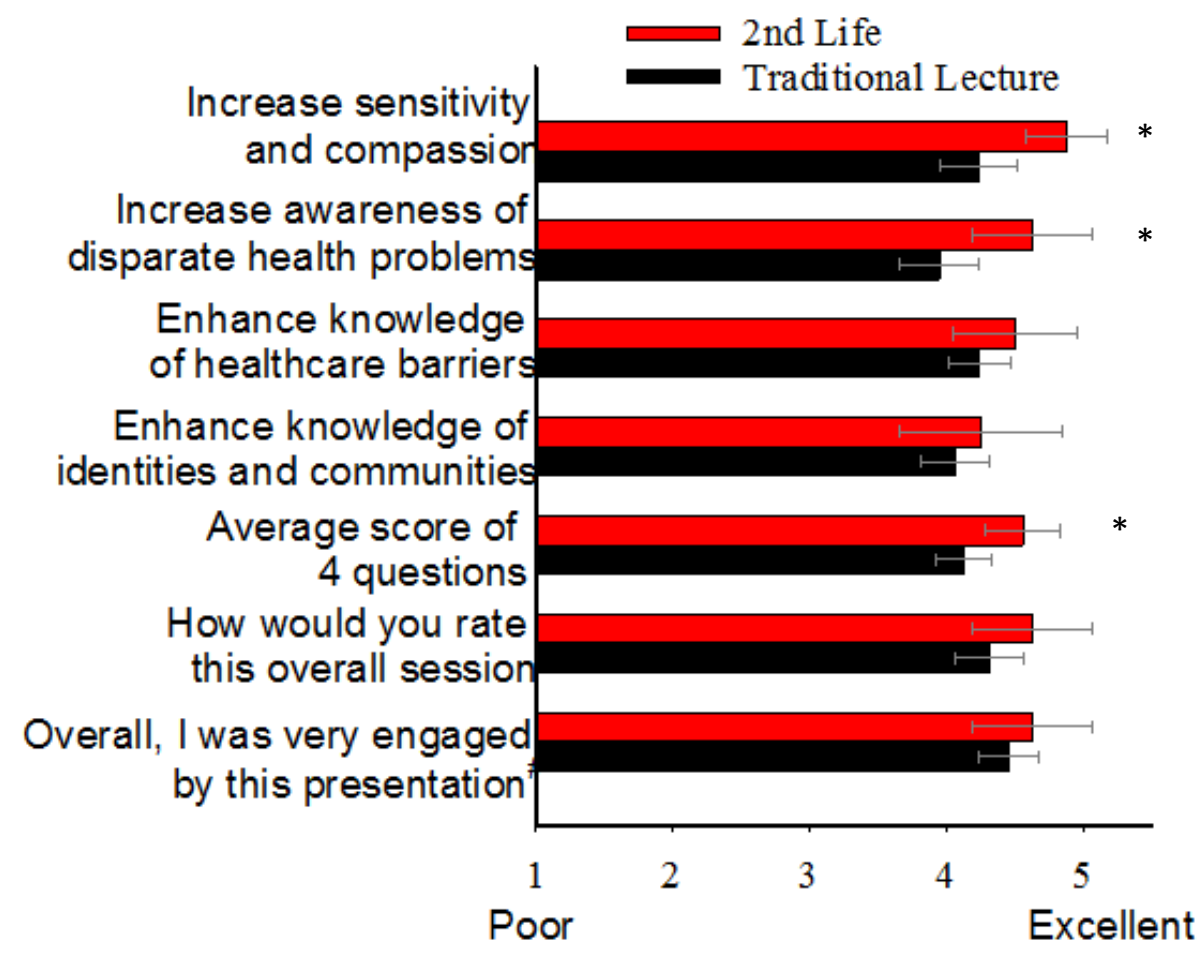

FIGURE 13: Post-session survey results: Graph depicting average rankings of the questions 2-6 and question 9. There was a significant difference between the Second Life ${ }^{\mathrm{TM}}$ and Traditional groups in assessing an increase in sensitivity and compassion and in assessing an increase in awareness of disparate health problems, Also, there was a significant difference found in the average score of questions the questions dealing with an increase in sensitivity and compassion, increase in awareness of disparate health problems, enhanced knowledge of healcare barriers, and enhanced knowledge of identities and communities. 


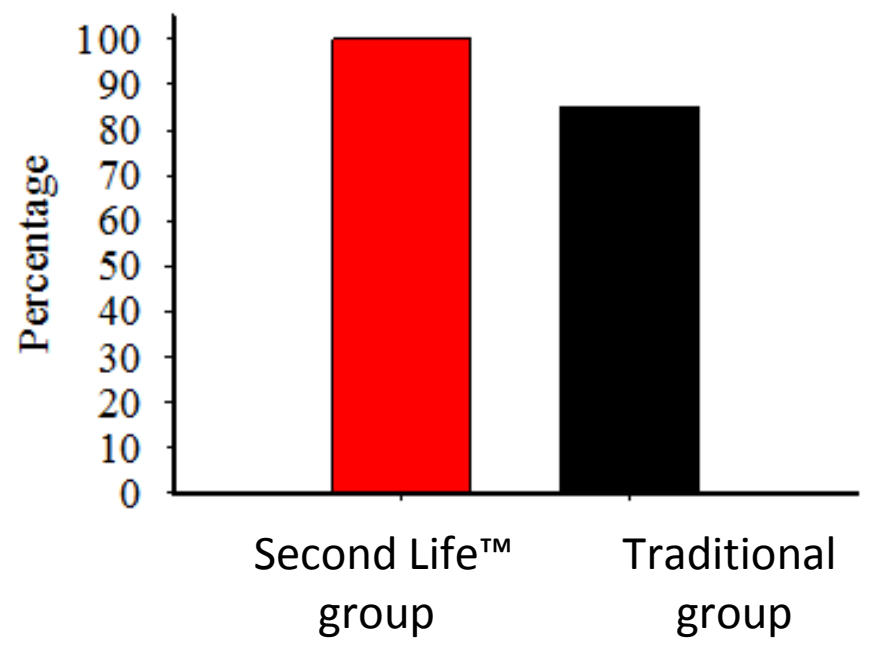

After this presentation, do you feel more confident working with this population?

FIGURE 14: Graph comparing the percent of students that felt more confident working with the LGBT population after attending the Second Life $^{\mathrm{TM}}$ workshop and the Traditional workshop. 


\begin{tabular}{|c|c|c|c|c|}
\hline & & $\mathrm{N}$ & Mean (SD) & Sig. \\
\hline \multirow{3}{*}{ Please rank-blanketexercise } & 2nd Life & 8 & $3.13(0.64)$ & \multirow{3}{*}{0.289} \\
\hline & & & & \\
\hline & Traditional Lecture & 39 & $2.62(1.11)$ & \\
\hline \multirow{3}{*}{ Please rank-scenarios } & 2nd Life & 8 & $3.13(1.13)$ & \multirow{3}{*}{0.153} \\
\hline & & & & \\
\hline & Traditional Lecture & 39 & $2.51(1.12)$ & \\
\hline \multirow{3}{*}{ Please rank-reflection } & 2nd Life & 8 & $2.13(1.25)$ & \multirow{3}{*}{0.109} \\
\hline & & & & \\
\hline & Traditional Lecture & 38 & $2.87(1.04)$ & \\
\hline \multirow{3}{*}{ Please rank-discussion of where to find resources/ongoing education } & 2nd Life & 8 & $2.00(1.07)$ & \multirow{3}{*}{0.746} \\
\hline & & & & \\
\hline & Traditional Lecture & 37 & $2.19(1.13)$ & \\
\hline
\end{tabular}

TABLE 1: Table comparing the average rankings of the four components of the workshops in the Second Life ${ }^{\mathrm{TM}}$ workshops and the Traditional Workshop. 


\begin{tabular}{|c|c|c|c|c|c|c|c|c|c|}
\hline & & $\begin{array}{c}\text { Poor } \\
\text { Freq }(\%)\end{array}$ & $\begin{array}{c}2 \\
\text { Freq }(\%)\end{array}$ & $\begin{array}{c}3 \\
\text { Freq }(\%)\end{array}$ & $\begin{array}{c}4 \\
\text { Freq }(\%)\end{array}$ & $\begin{array}{l}\text { Excellent } \\
\text { Freq }(\%)\end{array}$ & Mean & $(\mathrm{SD})$ & Sig. \\
\hline \multirow{3}{*}{$\begin{array}{l}\text { Did this session increase your sensitivity and } \\
\text { compassion for LGBTQ patients? }\end{array}$} & 2nd Life & $0(0)$ & $0(0)$ & $0(0)$ & $1(13)$ & $7(88)$ & 4.88 & $(0.35)$ & \multirow{3}{*}{0.05} \\
\hline & Traditional & & & & & & & & \\
\hline & Lecture & $1(2)$ & $0(0)$ & 7 (17) & $13(32)$ & $20(49)$ & 4.24 & $(0.92)$ & \\
\hline \multirow{3}{*}{$\begin{array}{l}\text { Did this session increase your awareness of } \\
\text { disparate health problems within this } \\
\text { population? }\end{array}$} & 2nd Life & $0(0)$ & $0(0)$ & $0(0)$ & $3(38)$ & $5(63)$ & 4.63 & $(0.52)$ & \multirow{3}{*}{0.05} \\
\hline & Traditional & & & & & & & & \\
\hline & Lecture & $1(2)$ & $1(2)$ & $9(22)$ & $18(44)$ & $12(29)$ & 3.95 & $(0.92)$ & \\
\hline \multirow{3}{*}{$\begin{array}{l}\text { Did this session enhance your knowledge of } \\
\text { bariers to healthcare specific to this } \\
\text { population? }\end{array}$} & 2nd Life & $0(0)$ & $0(0)$ & $0(0)$ & $4(50)$ & $4(50)$ & 4.50 & $(0.53)$ & \multirow{3}{*}{0.50} \\
\hline & Traditional & & & & & & & & \\
\hline & Lecture & $0(0)$ & $0(0)$ & 7 (17) & $17(41)$ & $17(41)$ & 4.24 & $(0.73)$ & \\
\hline \multirow{3}{*}{$\begin{array}{l}\text { Did this session enhance your knowledge of } \\
\text { LGBTQ identities and community? }\end{array}$} & 2nd Life & $0(0)$ & $0(0)$ & $1(13)$ & $4(50)$ & $3(38)$ & 4.25 & $(0.71)$ & \multirow{3}{*}{0.64} \\
\hline & Traditional & & & & & & & & \\
\hline & Lecture & $0(0)$ & $1(2)$ & $9(22)$ & $17(41)$ & $14(34)$ & 4.07 & $(0.82)$ & \\
\hline \multirow{3}{*}{ How would you rate this session overall? } & 2nd Life & $0(0)$ & $0(0)$ & $0(0)$ & $3(38)$ & $5(63)$ & 4.63 & $(0.52)$ & \multirow{3}{*}{0.35} \\
\hline & Traditional & & & & & & & & \\
\hline & Lecture & $1(2)$ & $0(0)$ & $2(5)$ & $20(49)$ & $18(44)$ & 4.32 & $(0.79)$ & \\
\hline \multirow{5}{*}{ Overall, I was very engaged by this presentation } & & Strongly & & & & Strongly & & & \multirow{5}{*}{0.75} \\
\hline & & disagree & 2 & 3 & 4 & agree & & & \\
\hline & 2nd Life & $0(0)$ & $0(0)$ & $0(0)$ & $3(38)$ & $5(63)$ & 4.63 & $(0.52)$ & \\
\hline & Traditional & & & & & & & & \\
\hline & Lecture & $0(0)$ & $1(2)$ & $2(5)$ & $15(37)$ & $23(56)$ & 4.46 & $(0.71)$ & \\
\hline \multirow{3}{*}{$\begin{array}{l}\text { After this presentation, do you feel more } \\
\text { confident working with this population? }\end{array}$} & & $\begin{array}{l}\text { No or } \\
\text { uncertain }\end{array}$ & Yes & & & & & & \multirow{3}{*}{0.57} \\
\hline & 2ndLife & $0(0)$ & $8(100)$ & & & & & & \\
\hline & Lecture & $6(15)$ & $35(85)$ & & & & & & \\
\hline
\end{tabular}

TABLE 2: Table comparing the frequency of ratings for questions 2-6, 9 of the post-survey. 


\section{CURRICULUM VITAE}

NAME: Ashley Mack

ADDRESS: 1559 South $25^{\text {th }}$ Street

Louisville, KY 40210

DOB: $\quad$ June 23, 1986

EDUCATION AND TRAINING:

B.S., Biology

Kentucky State University

2006-2011

\section{RESEARCH PRESENTATIONS:}

- 2013 Annual Meeting of the AAMC Southern Group on Educational Affairs

- University of Louisville Research Louisville Conference 2013

- 2011 U of I Summer Research Poster

- 2011 MANRRS National Convention Poster Competition: 2nd Place Winner

- 2009 \& 2011 Posters at the Capitol Presenter

- 2009 MANRRS National Convention Oral Competition Presenter 
ORGANIZATIONS/MEMBERSHIPS:

- Kentucky Academy of Sciences

- Delta Sigma Theta Sorority Inc.

- Minorities in Agriculture, Natural Resources and Related Sciences (MANRRS)

- Pre-Health Professionals Club

- American Association of University Women (AAUW)

- Ophthalmology Club-University of Louisville

COMMUNITY SERVICE:

- Campus Clean-up Initiative

- H.A.L.O.

- Center for Women and Families

- Habitat for Humanity

- Everybody Reads Program

- Reforest Frankfort

- Nation Day of Service

- Voter Registration

- Bone Marrow Donor Drive

- Bates Bookstore Volunteer

- Shepherdsville Elementary School- Free Back to School Vision Tests

HONORS AND AWARDS:

- National Honor Roll 2004

- National Latin Honor Society 2004

- 2008 Thurgood Marshall Scholar

- Dean's List 2008

- Posters at the Capitol

- Voter Registration

- 2010 MANRRS Research Poster Competition 2nd Place

- Dean's List 2010

- Dean's List 2011 